\title{
Integrated Systems Pharmacology and Surface Plasmon Resonance Approaches to Reveal the Synergistic Effect of Multiple Components of Gu-Ben- Ke-Chuan Decoction on Chronic Bronchitis
}

This article was published in the following Dove Press journal:

Journal of Inflammation Research

\begin{abstract}
Zhiqiang Luo',*
Guohua $\mathrm{Yu}^{\mathrm{l}}{ }^{\prime *}$

Wubin Wang ${ }^{1, *}$

Rui Sun'

Binbin Zhang'

Jing Wang ${ }^{2}$

Jing Liu'

Shan Gao'

Peng Wang ${ }^{3}$

Yuanyuan Shi ${ }^{1,4}$

'School of Life Sciences, Beijing University of Chinese Medicine, Beijing, 102488, People's Republic of China; ${ }^{2}$ State Key Laboratory of Natural and Biomimetic Drugs, School of Pharmaceutical Sciences, Peking University, Beijing, I00191, People's Republic of China; ${ }^{3}$ The First Affiliated Hospital of Guangzhou Medical University, Guangzhou, 510120, People's Republic of China; ${ }^{4}$ Shenzhen Research Institute, Beijing University of Chinese Medicine, Shenzhen, 518I 18, People's Republic of China

*These authors contributed equally to this work
\end{abstract}

Correspondence: Peng Wang

The First Affiliated Hospital of Guangzhou Medical University, No. I5I Yanjiang

Road, Yuexiu District, Guangzhou,

510120, People's Republic of China

Emailmrwp0022@163.com

Yuanyuan Shi

Shenzhen Research Institute, Beijing University of Chinese Medicine, No. 16 Lanjingzhong Road, Pingshan District, Shenzhen, 518118, People's Republic of China

Tel +86I8565780299

Email yshi@bucm.edu.cn
Introduction: Gu-Ben-Ke-Chuan (GBKC) decoction, a well-known prescription composed of seven herbs, has been widely used for treating chronic bronchitis (CB). However, the pharmacological constituents of GBKC and the underlying mechanisms by which these components act on $\mathrm{CB}$ remain unclear.

Methods: Ultra-high-pressure liquid chromatography coupled with linear ion trap-Orbitrap tandem mass spectrometry (UHPLC-LTQ-Orbitrap) was first employed to rapidly identify compounds from GBKC. Thereafter, network pharmacology and molecular docking analyses were performed to identify the potential active constituents, candidate targets, and major pathways. Finally, the affinities between the key compounds and targets were verified via surface plasmon resonance (SPR) analysis. In addition, the anti-inflammatory effect of GBKC was verified using an LPS-induced inflammatory cell model based on the predicted results.

Results: A total of 53 major compounds were identified in the GBKC decoction. After network pharmacology-based virtual screening, 141 major targets and 39 main compounds were identified to be effective in the treatment of $\mathrm{CB}$. The major targets were highly enriched in the tumor necrosis factor (TNF) signaling pathway, suggesting that GBKC could attenuate the inflammatory response in patients with CB. Furthermore, molecular docking results indicated that 20 pairs of components and target proteins relevant to the TNF pathway exhibited notable interactions. Among them, eight compound-target pairs exhibited good affinity as per SPR analysis. In addition, the production of interleukin 6 and TNF- $\alpha$ in LPSinduced MH-S cells was suppressed after GBKC treatment.

Conclusion: This study successfully clarified the mechanism of action of GBKC against $\mathrm{CB}$, which demonstrated that the integrated strategy described above is reliable for identifying the active compounds and mechanisms responsible for the pharmacological activities of GBKC decoction.

Keywords: Gu-Ben-Ke-Chuan decoction, chronic bronchitis, UHPLC-LTQ-Orbitrap, network pharmacology, surface plasmon resonance

\section{Introduction}

Chronic bronchitis (CB), a type of chronic obstructive pulmonary disease, is defined as chronic cough and sputum production for 3 months/year for two consecutive years. ${ }^{1} \mathrm{CB}$ remains the fourth largest cause of hospitalizations and death worldwide, and has brought a heavy health burden and soaring healthcare costs to 
individuals, families, and society. ${ }^{2}$ Many environmental and host factors may contribute to $\mathrm{CB}$ progression or exacerbation, such as tobacco smoke, infection, occupational hazards, and poor lung development during childhood. $^{3}$ Although the number of patients with $\mathrm{CB}$ keeps increasing, its pathophysiological mechanism is not yet clearly understood. Current pharmacological treatments for $\mathrm{CB}$, including antibiotics and corticosteroids, may compromise the efficacy and safety characteristics of these agents, resulting in unacceptable long-term adverse reactions, such as diarrhea, nausea, osteoporosis, pneumonia, and psychiatric disturbances.; ${ }^{4-6}$ therefore, new anti-CB medications with good efficacy and safety are urgently required.

Gu-Ben-Ke-Chuan (GBKC) decoction, a well-known traditional Chinese herbal formula, contains seven herbs such as Radix Codonopsis ("Dang-Shen" in Chinese, DS), Rhizoma Atractylodis Macrocephalae ("Bai-Zhu" in Chinese, BZ), Poria cocos ("Fu-Ling" in Chinese, FL), Radix Ophiopogonis ("Mai-Dong" in Chinese, MD), Fructus Psoraleae ("Bu-Gu-Zhi" in Chinese, BGZ), Glycyrrhizae Radix et Rhizoma ("Gan-Cao" in Chinese, GC), and Schisandrae Chinensis Fructus ("Wu-Wei-Zi" in Chinese, WWZ) at the weight ratio of 6:6:4:6:6:3:3. This traditional Chinese medicine (TCM) prescription has been recorded in the Chinese Pharmacopeia and approved by the China Food and Drug Administration for the treatment of airway diseases, such as asthma, $\mathrm{CB}$, and bronchiectasis. ${ }^{7}$ In recent years, accumulating evidence has demonstrated that GBKC exerts substantial therapeutic effects on $\mathrm{CB}{ }^{8-10}$ However, its underlying mechanisms of action are still largely unknown.

As a relatively new discipline in modern pharmacological studies, network pharmacology has been proposed as a promising approach to obtain a systematic understanding of the complex relationships between drug and disease, which adheres to the holistic philosophy of traditional Chinese medicine (TCM). ${ }^{11}$ To date, this method has been successfully used to reveal the synergistic effects and molecular mechanisms of TCM in many complicated diseases, such as Alzheimer's disease, ${ }^{11}$ cardiovascular disease, ${ }^{12,13}$ diabetes, ${ }^{14}$ uveitis, ${ }^{15}$ cancer, ${ }^{16}$ and gastrointestinal diseases. ${ }^{17}$

Surface plasmon resonance (SPR) biosensor serves as a powerful tool for label-free and real-time analysis of the interaction between drug and protein; ${ }^{18,19}$ it can quickly and easily monitor the association and disassociation of molecules. ${ }^{20}$ In addition, SPR can directly screen active compounds from complex matrices. $^{21}$ Consequently, optical biosensor methods based on SPR have been extensively used in TCM research, such as for lead compound discovery and target identification. ${ }^{22}$

In this study, the main chemical compounds were first detected and identified using UHPLC-LTQOrbitrap. Second, a network pharmacology-based strategy was used to explore the active components and major targets of GBKC for treating CB. Third, highthroughput molecular docking studies using LibDock were performed to confirm the binding activities between the active components and the related targets. Fourth, the specific interactions between the targets and key components were investigated and verified using SPR methodologies. Finally, the anti-inflammatory function of GBKC was further verified via in vitro cell experiments based on the predicted results. We hope that this study will not only reveal the anti-CB mechanism of GBKC, but also provide a rapid and reliable strategy for screening active compounds from medicinal plants and prescriptions. The workflow of this study is illustrated in Figure 1.

\section{Materials and Methods Materials and Reagents}

The seven herbs, including Radix Codonopsis, Rhizoma Atractylodis Macrocephalae, Poria cocos, Radix Ophiopogonis, Fructus Psoraleae, Glycyrrhizae Radix et Rhizoma, and Schisandrae Chinensis Fructus were purchased from Beijing Tongrentang and authenticated by Professor Jingjuan Wang (Beijing University of Chinese Medicine). Chromatographic-grade acetonitrile was supplied by Fisher Scientific. Ultrapure water was prepared using the Millipore Milli Q plus purification system. All other reagents used were of analytical grade and commercially available.

The mouse alveolar macrophage cell line MH-S was purchased from Peking Union Medical College (Beijing, China). RPMI-1640 culture medium and trypsin were provided by Corning Inc. (New York, NY, USA). Fetal bovine serum (FBS) and a mixture of penicillin and streptomycin were purchased from Gibco (Grand Island, NY, USA). Lipopolysaccharide (Escherichia coli 055:B5, LPS) was purchased from Yuanye BioTechnology Co., Ltd. (Shanghai, China). Cell Counting 


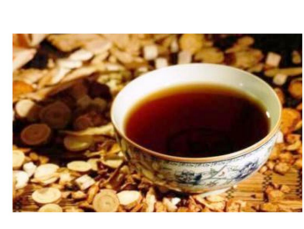

GBKC decoction

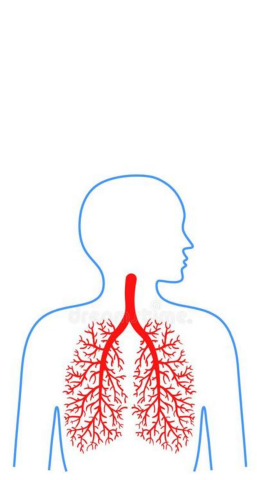

CB

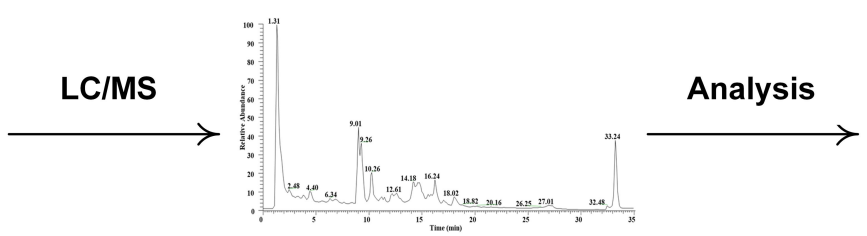

TIC

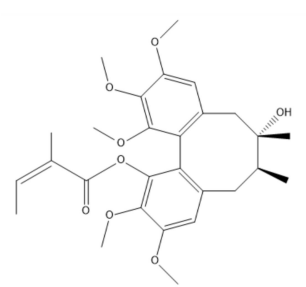

GBKC ingredients

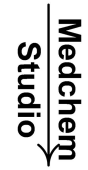

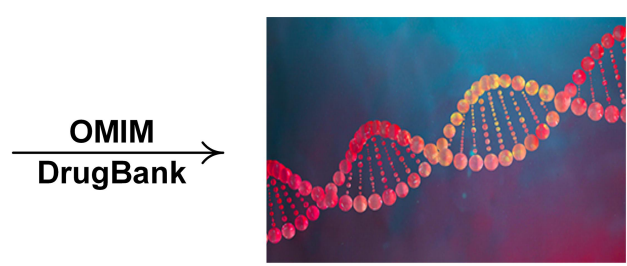

CB-associated genes

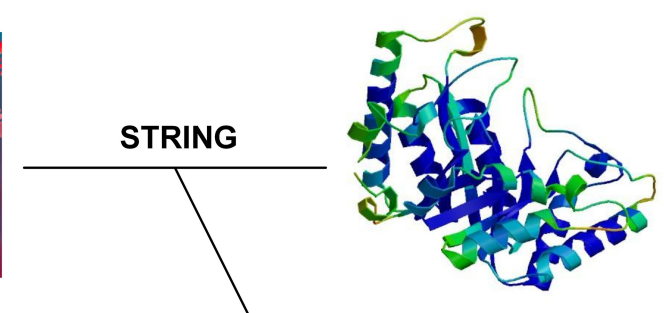

Targets of GBKC

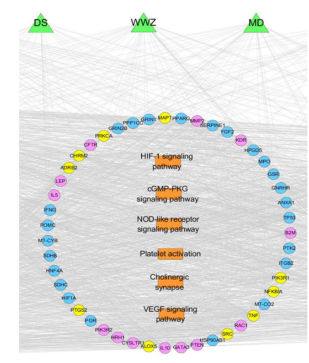

Key network

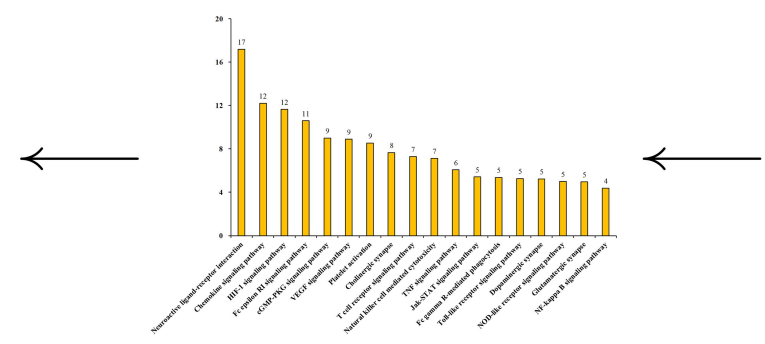

GO\&KEGG performance

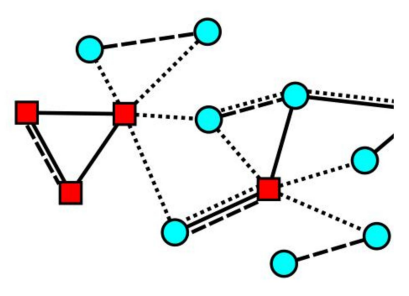

Network analysis

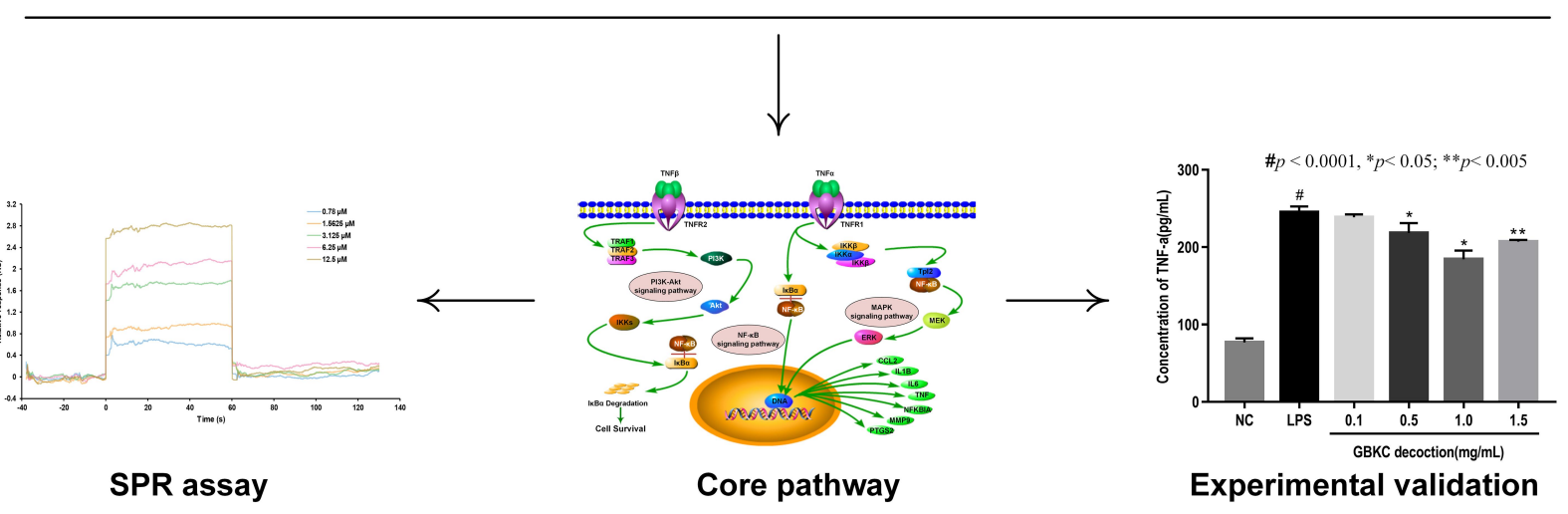

Figure I Experimental design and schematic diagram of the workflow of this study. 
Kit-8 (CCK-8) was purchased from KeyGEN BioTechnology Co., Ltd. (Nanjing, China). Enzyme-linked immunosorbent assay (ELISA) kit for TNF- $\alpha$ and IL-6 were purchased from Proteintech Group, Inc. (Chicago, IL, USA).

\section{Preparation of GBKC Decoction}

DS (30g), BZ (30g), FL (20g), MD (30g), BGZ (30g), GC $(15 \mathrm{~g})$ and WWZ $(15 \mathrm{~g})$ were mixed and immersed with six-fold mass of water for $1 \mathrm{~h}$. The mixtures were boiled twice, $1 \mathrm{~h}$ for each time. The extracted water solutions were combined, filtered, and concentrated to $2 \mathrm{~g} / \mathrm{mL}$, and stored at $-20^{\circ} \mathrm{C}$ until use. For chemical analysis, the GBKC decoction $(2 \mathrm{~g} / \mathrm{mL})$ was diluted to $10 \mathrm{mg} / \mathrm{mL}$ (w/v, crude drug/water) and filtered using a $0.22 \mu \mathrm{m}$ membrane filter before UHPLC-LTQOrbitrap analysis. For cell experiments, the GBKC decoction $(2 \mathrm{~g} / \mathrm{mL})$ was sterilized and diluted to appointed concentrations $(0.1,0.5,1.0,1.5 \mathrm{mg} / \mathrm{mL})$ with cell culture medium.

\section{LC/MS Analysis}

For LC/MS experiments, an LTQ-Orbitrap Velos mass spectrometer equipped with an electrospray ionization (ESI) source was connected to a Thermo Dionex Ultimate 3000 UHPLC system (including an auto-sampler, a thermostatically controlled column compartment and a binary pump coupled to a vacuum degasser). The Xcalibur, Metworks and Mass Frontier 6.0 software packages (Thermo Fisher Scientific) were employed for data recording and processing.

LC analysis was conducted on a Thermo Scientific Hypersil BDS $\mathrm{C}_{18}(2.1 \mathrm{~mm} \times 150 \mathrm{~mm}, 2.4 \mu \mathrm{m})$ column maintained at $35^{\circ} \mathrm{C}$. The mobile phase system was composed of water with $0.1 \%$ formic acid (A) and acetonitrile (B). The flow rate was set to $0.3 \mathrm{~mL} / \mathrm{min}$ with a linear gradient as follows: $0-6.0 \mathrm{~min}(3-3 \% \mathrm{~B}), 6.0-13.0 \mathrm{~min}$ (3-15\% B), 13.0-23.0 min (15-40\% B), 23.0-25.0 min $(40-65 \%$ B), $25.0-30.0 \min (65-97 \%$ B), 30.0-30.5 $(97-3 \% \mathrm{~B})$ and $30.5-35.0(3-3 \% \mathrm{~B})$. The injection volume was $3 \mu \mathrm{L}$.

The MS analysis was conducted on both the negative and positive ion modes. The capillary temperature was $350{ }^{\circ} \mathrm{C}$, source voltage was set at $3.0 \mathrm{kV}$, sheath gas $\left(\mathrm{N}_{2}\right)$ flow was $40 \mathrm{Arb}$ and aux gas flow rate was $20 \mathrm{Arb}$. Full MS scans were acquired in the range of $\mathrm{m} / \mathrm{z}$ 50-1500 and the MS/MS experiments were set as datadependent scans.

\section{Predicting the Targets of the Identified Components Within GBKC}

MedChem Studio (MedChem Studio, 3.0; Simulations Plus, Inc., Lancaster, CA, USA, 2012), a powerful tool for predicting targets based on structure similarity, was applied to retrieve the targets of candidate ingredients of GBKC, with the similarity threshold set at $0.6 .^{23}$

\section{Known Therapeutic Targets Associated with $C B$}

The target proteins/genes associated with FDA-approved drugs for $\mathrm{CB}$ were retrieved from DrugBank database (http://www.drugbank.ca/, version 5.1.1) and Online Mendelian Inheritance in Man (OMIM) database (http:// www.omim.org/, updated on May 4, 2018) using the keyword "Chronic bronchitis" and confining the species to "Homo sapiens." DrugBank is a high quality database containing comprehensive information on drugs and their targets. ${ }^{24}$ OMIM is a comprehensive, freely accessible database of genetic diseases and their related human genes. $^{25}$

\section{Protein-Protein Interaction (PPI) Data}

PPI data were extracted from Search Tool for the Retrieval of Interacting Genes/Proteins (STRING) database (http://string-db.org/) which integrated almost all publicly available sources of PPI information. ${ }^{26}$ The cut-off value was set at 0.4 for the screening of high confidence edges.

\section{Network Construction and Analysis}

For understanding the relationships between the predicted targets of GBKC and CB-related target genes/ proteins, Cytoscape (version 3.6.0, Boston, MA, USA) was adopted to visualize the "bioactive componentstargets-disease" network and draw the network diagram. Using the Cytoscape option network analyzer, the degree values of nodes in the network were assessed, and a node was represented as a hub when its degree value was two-fold above the median value of all nodes in the network. Next, the hub network was constructed by employing direct interactions among the hubs. Moreover, to estimate the topological importance of the hub nodes, "degree," "betweenness," and "closeness" were calculated. According to our previous studies, ${ }^{11}$ if the values of the three topological 
properties exceeded the corresponding median values, these hub nodes would be recognized as the key GBKC targets for CB.

\section{Pathway Enrichment Analysis}

To determine the significance of the major hubs, the Database for Annotation, Visualization and Integrated Discovery Bioinformatics Resources 6.7 online tool (http://david.abcc.ncifcrf.gov/home.jsp/, version 6.7) was employed to analyze the Kyoto Encyclopedia of Genes and Genomes (KEGG) pathway (http://www.gen ome.jp/kegg/) and gene ontology (GO) enrichment of GBKC on the direct or indirect targets of $\mathrm{CB}$. The threshold value of confident gene enrichment was set at $P<0.05$.

\section{Molecular Docking Stimulation}

Molecular docking studies were carried out to validate the credibility of the compound-target interactions using the Discovery Studio 2016 option LibDock. The high-resolution crystal structures of the key targets were extracted from the RCSB protein database (http://www.pdb.org/) and prepared by deleting the co-crystallized ligand and water molecules, adding hydrogen atoms, and fixing the side chains. The binding site was defined by the ligand atoms, and the cutoff radius was set to $10 \AA$. Chem3D Pro 12.0 was used to generate the three-dimensional structures of the candidate components that were saved in PDB format. The docking scores were used to evaluate the binding affinities between the hub targets and their corresponding compounds. A docked conformation with the best fitness score was selected. The other parameters used in calculation were default except for explained.

\section{SPR Analysis}

Based on the virtual screening results, four target proteins were selected for SPR analysis: nuclear factor kappa B (NFKB1, Novoprotein), matrix metalloproteinase-9 (MMP-9, Novoprotein), tumor necrosis factor (TNF- $\alpha$, Novoprotein), and mitogen-activated protein kinase (MAPK1, Sino Biological). SPR experiments were performed using a Biacore $8 \mathrm{~K}$ (GE Healthcare, Sweden). The surface of the CM5 sensor chip was activated by injecting a freshly prepared mixture of NHS and EDC (1:1, v/v). NFKB1, MMP-9,
TNF- $\alpha$, and MAPK1 were immobilized on CM5 chips via a standard amine coupling method with immobilization levels of $11,800,14,874,11,000$, and 11,000 response units, respectively. Detection was conducted according to the protocol supplied by GE Healthcare. For each sample analysis, a reference channel without the conjugated protein was activated and blocked to eliminate non-specific binding to the sensor surface. The analytes were injected at a flow rate of $30 \mu \mathrm{L} /$ min. The association and dissociation times were set at $60 \mathrm{~s}$ and $120 \mathrm{~s}$, respectively. The SPR curves were fitted using Biacore Insight Evaluation Software according to the 1:1 Langmuir binding model and the steady-state affinity model, from which the kinetics (the association rate constant, $\mathrm{K}_{\mathrm{a}}$; the dissociation rate constant, $\left.\mathrm{K}_{\mathrm{d}}\right)$ and affinity constants $\left(\mathrm{K}_{\mathrm{D}}\right)$ were calculated.

\section{Cell Culture and Treatment}

The MH-S cells were maintained in RPMI-1640 medium containing $10 \% \mathrm{FBS}$ and $1 \%$ penicillin-streptomycin at $37{ }^{\circ} \mathrm{C}$ in $5 \% \mathrm{CO}_{2}$ humidified air. For each experiment, the cells were stimulated with LPS $(1 \mu \mathrm{g} / \mathrm{mL})$ in the presence or absence of $\operatorname{GBKC}(0.1,0.5,1.0$, and $1.5 \mathrm{mg} / \mathrm{mL})$ for $24 \mathrm{~h}$.

\section{Cell Viability Assay}

A cell counting Kit-8 (CCK-8) assay was performed to assess the viability of $\mathrm{MH}-\mathrm{S}$ cells after GBKC treatment. Briefly, the cells were seeded into 96-well plates at a density of $3 \times 10^{4}$ cells/well and then treated with or without GBKC $(0.1,0.5,1.0$, or $1.5 \mathrm{mg} / \mathrm{mL})$ for $24 \mathrm{~h}$. Next, cell-free supernatants were removed and $200 \mu \mathrm{L}$ of $10 \%$ CCK- 8 solution was added to each well. The plates were then incubated at $37^{\circ} \mathrm{C}$ for another $0.5-2 \mathrm{~h}$. Finally, the optical density of each well was measured at $490 \mathrm{~nm}$ using a microplate reader (Molecular Devices, Sunnyvale, CA, USA).

\section{Enzyme-Linked Immunosorbent Assay (ELISA)}

The levels of TNF- $\alpha$ and interleukin (IL)-6, two vital cytokines in the early stage of inflammation, ${ }^{27}$ in the cell incubation medium were determined using ELISA kits following the manufacturer's instructions. 
Table I Analysis of the Chemical Constituents of GBKC by UHPLC-LTQ-Orbitrap in Positive lon Mode

\begin{tabular}{|c|c|c|c|c|c|c|c|}
\hline No. & $\begin{array}{c}\text { RT } \\
\text { (Min) }\end{array}$ & $\begin{array}{l}{\left[\mathrm{M}+\mathrm{H}^{+} /\right.} \\
{[\mathrm{M}+\mathrm{Na}]^{+}}\end{array}$ & MS/MS & $\begin{array}{l}\text { Molecular } \\
\text { Formula }\end{array}$ & $\begin{array}{l}\text { Error } \\
(\mathrm{ppm})\end{array}$ & Compound & Herb \\
\hline 3 & 5.81 & 268.1534 & $88.0755\left[\mathrm{C}_{4} \mathrm{H}_{10} \mathrm{ON}\right]^{+}$ & $\mathrm{C}_{14} \mathrm{H}_{22} \mathrm{O}_{4} \mathrm{~N}$ & -0.955 & Codonopsine & DS \\
\hline 4 & 8.79 & 565.1530 & $547.1432\left[\mathrm{M}+\mathrm{H}-\mathrm{H}_{2} \mathrm{O}\right]^{+}, 529.1332\left[\mathrm{M}+\mathrm{H}-2 \mathrm{H}_{2} \mathrm{O}\right]^{+}$ & $\mathrm{C}_{26} \mathrm{H}_{28} \mathrm{O}_{14}$ & -3.949 & Schaftoside/lsoschaftoside & GC \\
\hline 5 & 9.01 & 389.0830 & $\begin{array}{l}345.0941\left[\mathrm{M}+\mathrm{Na}-\mathrm{CO}_{2}\right]^{+}, 227.031 \mathrm{I}[\mathrm{M}+\mathrm{Na}- \\
\left.\mathrm{C}_{6} \mathrm{H}_{10} \mathrm{O}_{5}\right]^{+}, 185.0417\left[\mathrm{C}_{6} \mathrm{H}_{10} \mathrm{O}_{5} \mathrm{Na}\right]^{+}\end{array}$ & $\mathrm{C}_{17} \mathrm{H}_{18} \mathrm{O}_{9}$ & -3.401 & $\begin{array}{l}\text { Psoralenoside/ } \\
\text { Isopsoralenoside }\end{array}$ & BGZ \\
\hline 6 & 9.26 & 389.0828 & $\begin{array}{l}345.0940\left[\mathrm{M}+\mathrm{Na}-\mathrm{CO}_{2}\right]^{+}, 227.0311[\mathrm{M}+\mathrm{Na}- \\
\left.\mathrm{C}_{6} \mathrm{H}_{10} \mathrm{O}_{5}\right]^{+}, 185.0417\left[\mathrm{C}_{6} \mathrm{H}_{10} \mathrm{O}_{5} \mathrm{Na}\right]^{+}\end{array}$ & $\mathrm{C}_{17} \mathrm{H}_{18} \mathrm{O}_{9}$ & -3.966 & $\begin{array}{l}\text { Psoralenoside/ } \\
\text { Isopsoralenoside }\end{array}$ & BGZ \\
\hline 7 & 9.54 & 565.1535 & $547.1439\left[\mathrm{M}+\mathrm{H}-\mathrm{H}_{2} \mathrm{O}\right]^{+}, 529.1339\left[\mathrm{M}+\mathrm{H}-2 \mathrm{H}_{2} \mathrm{O}\right]^{+}$ & $\mathrm{C}_{26} \mathrm{H}_{28} \mathrm{O}_{14}$ & -2.976 & Schaftoside/lsoschaftoside & GC \\
\hline 9 & 10.32 & 257.0801 & $\begin{array}{l}242.0570\left[\mathrm{M}+\mathrm{H}-\mathrm{CH}_{3}\right]^{+}, 239.070 \mathrm{I}\left[\mathrm{M}+\mathrm{H}-\mathrm{H}_{2} \mathrm{O}\right]^{+}, \\
21 \mathrm{I} .075 \mathrm{I}\left[\mathrm{M}+\mathrm{H}-\mathrm{H}_{2} \mathrm{O}-\mathrm{CO}\right]^{+}, \mathrm{I} 63.0387[\mathrm{M} \\
\left.+\mathrm{H}-\mathrm{H}_{2} \mathrm{O}-\mathrm{C}_{6} \mathrm{H}_{4}\right]^{+}, \mathrm{I} 47.0437\left[\mathrm{M}+\mathrm{H}-\mathrm{C}_{6} \mathrm{H}_{6} \mathrm{O}_{2}\right]^{+}, \\
137.0230\left[\mathrm{M}+\mathrm{H}-\mathrm{C}_{8} \mathrm{H}_{8} \mathrm{O}\right]^{+}, \mathrm{I} 19.0489\left[\mathrm{C}_{8} \mathrm{H}_{7} \mathrm{O}\right]^{+}\end{array}$ & $\mathrm{C}_{15} \mathrm{H}_{12} \mathrm{O}_{4}$ & -2.938 & Liquiritigenin & GC \\
\hline 10 & 10.39 & 551.1738 & $\begin{array}{l}419.1330\left[\mathrm{M}+\mathrm{H}-\mathrm{C}_{5} \mathrm{H}_{8} \mathrm{O}_{4}\right]^{+}, 389.1225[\mathrm{M} \\
\left.+\mathrm{H}_{-} \mathrm{C}_{6} \mathrm{H}_{10} \mathrm{O}_{5}\right]^{+}, 257.0806\left[\mathrm{M}+\mathrm{H}-\mathrm{C}_{5} \mathrm{H}_{8} \mathrm{O}_{4}^{-}\right. \\
\left.\mathrm{C}_{4} \mathrm{H}_{6} \mathrm{O}_{4}-\mathrm{C}_{2} \mathrm{H}_{2} \mathrm{O}\right]^{+}\end{array}$ & $\mathrm{C}_{26} \mathrm{H}_{30} \mathrm{O}_{13}$ & -3.787 & Liquiritin apioside & GC \\
\hline 11 & 10.42 & 257.0801 & $\begin{array}{l}242.0577\left[\mathrm{M}+\mathrm{H}-\mathrm{CH}_{3}\right]^{+}, 239.0707\left[\mathrm{M}+\mathrm{H}-\mathrm{H}_{2} \mathrm{O}\right]^{+} \\
211.0757\left[\mathrm{M}+\mathrm{H}-\mathrm{CH}_{2} \mathrm{O}_{2}\right]^{+}, \quad 147.0442[\mathrm{M} \\
\left.+\mathrm{H}-\mathrm{C}_{6} \mathrm{H}_{6} \mathrm{O}_{2}\right]^{+}, \quad 137.0233\left[\mathrm{M}+\mathrm{H}-\mathrm{C}_{8} \mathrm{H}_{8} \mathrm{O}\right]^{+}\end{array}$ & $\mathrm{C}_{15} \mathrm{H}_{12} \mathrm{O}_{4}$ & -2.938 & Isoliquiritigenin & GC \\
\hline 14 & 12.11 & 551.1738 & $\begin{array}{l}419.1330\left[\mathrm{M}+\mathrm{H}-\mathrm{C}_{5} \mathrm{H}_{8} \mathrm{O}_{4}\right]^{+}, 389.1225[\mathrm{M} \\
\left.+\mathrm{H}-\mathrm{C}_{6} \mathrm{H}_{10} \mathrm{O}_{5}\right]^{+}, 257.0806\left[\mathrm{M}+\mathrm{H}-\mathrm{C}_{5} \mathrm{H}_{8} \mathrm{O}_{4-}^{-}\right. \\
\left.\mathrm{C}_{4} \mathrm{H}_{6} \mathrm{O}_{4}-\mathrm{C}_{2} \mathrm{H}_{2} \mathrm{O}\right]^{+}\end{array}$ & $\mathrm{C}_{26} \mathrm{H}_{30} \mathrm{O}_{13}$ & -3.787 & Liquiritin apioside isomer & GC \\
\hline 15 & 12.44 & 419.1331 & $257.0805\left[\mathrm{M}+\mathrm{H}-\mathrm{C}_{6} \mathrm{H}_{10} \mathrm{O}_{5}\right]^{+}$ & $\mathrm{C}_{21} \mathrm{H}_{22} \mathrm{O}_{9}$ & -1.285 & Isoliquiritin & GC \\
\hline 16 & 12.59 & 431.132 & NA & $\mathrm{C}_{22} \mathrm{H}_{22} \mathrm{O}_{9}$ & -3.801 & Ononin & GC \\
\hline 17 & 12.59 & 269.0800 & $\begin{array}{l}254.0569\left[\mathrm{M}+\mathrm{H}-\mathrm{CH}_{3}\right]^{+}, 237.0544[\mathrm{M} \\
\left.+\mathrm{H}-\mathrm{CH}_{3} \mathrm{OH}\right]^{+}, 213.0907[\mathrm{M}+\mathrm{H}-2 \mathrm{CO}]^{+}\end{array}$ & $\mathrm{C}_{16} \mathrm{H}_{12} \mathrm{O}_{4}$ & -3.476 & Formononetin & GC \\
\hline 18 & 14.10 & 350.1950 & $\begin{array}{l}250.1435\left[\mathrm{M}-\mathrm{C}_{5} \mathrm{H}_{8} \mathrm{O}_{2}\right]^{+}, 220.1329\left[\mathrm{M}-\mathrm{C}_{6} \mathrm{H}_{10} \mathrm{O}_{3}\right]^{+} \text {, } \\
182.1174\left[\mathrm{M}-\mathrm{C}_{5} \mathrm{H}_{8} \mathrm{O}_{2}-\mathrm{C}_{4} \mathrm{H}_{4} \mathrm{O}\right]^{+}, 205.0856 \\
{\left[\mathrm{M}-\mathrm{C}_{5} \mathrm{H}_{8} \mathrm{O}_{2}-\mathrm{C}_{2} \mathrm{H}_{7} \mathrm{~N}\right]^{+}, \mathrm{I} 1 \mathrm{I} .0595\left[\mathrm{C}_{10} \mathrm{H}_{9} \mathrm{O}_{2}\right]^{+}}\end{array}$ & $\mathrm{C}_{19} \mathrm{H}_{28} \mathrm{O}_{5} \mathrm{~N}$ & -0.669 & Codonopyrrolidium A & DS \\
\hline 19 & 14.25 & 187.0382 & $\begin{array}{l}\left.\text { I59.0438[M+H-H } \mathrm{H}_{2} \mathrm{O}\right]^{+}, \text {I } 43.0487\left[\mathrm{M}+\mathrm{H}-\mathrm{CO}_{2}\right]^{+} \\
\text {I } 31.0488\left[\mathrm{M}+\mathrm{H}-\mathrm{C}_{2} \mathrm{O}_{2}\right]^{+}, \mathrm{I} / 5.0539\left[\mathrm{M}+\mathrm{H}-\mathrm{C}_{2} \mathrm{O}_{3}\right]^{+}\end{array}$ & $\mathrm{C}_{11} \mathrm{H}_{6} \mathrm{O}_{3}$ & -4.013 & Psoralen/Angelicin & BGZ \\
\hline 20 & 14.55 & 187.0382 & $\begin{array}{l}\mathrm{I} 59.0439\left[\mathrm{M}+\mathrm{H}-\mathrm{H}_{2} \mathrm{O}\right]^{+}, \mathrm{I} 43.0488[\mathrm{M} \\
\left.+\mathrm{H}-\mathrm{CO}_{2}\right]^{+}, \mathrm{I} 3 \mathrm{I} .0489\left[\mathrm{M}+\mathrm{H}-\mathrm{C}_{2} \mathrm{O}_{2}\right]^{+}, \mathrm{I} 15.0540 \\
{\left[\mathrm{M}+\mathrm{H}-\mathrm{C}_{2} \mathrm{O}_{3}\right]^{+}}\end{array}$ & $\mathrm{C}_{1}, \mathrm{H}_{6} \mathrm{O}_{3}$ & -4.013 & Psoralen/Angelicin & BGZ \\
\hline 21 & $14.7 \mid$ & 469.3296 & $45 \mathrm{I} .3203\left[\mathrm{M}+\mathrm{H}-\mathrm{H}_{2} \mathrm{O}\right]^{+}, 433.3100\left[\mathrm{M}+\mathrm{H}-2 \mathrm{H}_{2} \mathrm{O}\right]^{+}$ & $\mathrm{C}_{30} \mathrm{H}_{44} \mathrm{O}_{4}$ & -3.444 & 16-deoxyporicoic acid B & $\mathrm{FL}$ \\
\hline 22 & 14.77 & 839.4041 & NA & $\mathrm{C}_{42} \mathrm{H}_{62} \mathrm{O}_{17}$ & -2.224 & Licoricesaponin G2/isomer & GC \\
\hline 23 & 15.58 & 839.4041 & NA & $\mathrm{C}_{42} \mathrm{H}_{62} \mathrm{O}_{17}$ & -2.224 & Licoricesaponin G2/isomer & GC \\
\hline 25 & 15.60 & 337.1062 & $319.0961\left[\mathrm{M}+\mathrm{H}-\mathrm{H}_{2} \mathrm{O}\right]^{+}, 279.0650\left[\mathrm{M}+\mathrm{H}-\mathrm{C}_{3} \mathrm{H}_{6} \mathrm{O}\right]^{+}$ & $\mathrm{C}_{20} \mathrm{H}_{16} \mathrm{O}_{5}$ & -2.433 & Psoralidin & BGZ \\
\hline 28 & 16.93 & 777.4008 & NA & $\mathrm{C}_{39} \mathrm{H}_{62} \mathrm{O}_{14}$ & -3.110 & $\begin{array}{l}\text { (25R)-spirost-5-ene- } \\
3 \beta,|4 \alpha,| 7 \alpha \text {-triol-3-O- } \alpha \text { - } \\
\text { L-rhamnopyranosyl-(I } \rightarrow 2)-\beta- \\
\text { D-glucopyranoside }\end{array}$ & MD \\
\hline
\end{tabular}

(Continued) 
Table I (Continued).

\begin{tabular}{|c|c|c|c|c|c|c|c|}
\hline No. & $\begin{array}{c}\text { RT } \\
\text { (Min) }\end{array}$ & $\begin{array}{l}{[\mathrm{M}+\mathrm{H}]^{+} /} \\
{[\mathrm{M}+\mathrm{Na}]^{+}}\end{array}$ & MS/MS & $\begin{array}{l}\text { Molecular } \\
\text { Formula }\end{array}$ & $\begin{array}{l}\text { Error } \\
(\mathrm{ppm})\end{array}$ & Compound & Herb \\
\hline 30 & 17.30 & 985.4611 & NA & $\mathrm{C}_{48} \mathrm{H}_{72} \mathrm{O}_{21}$ & -2.806 & Licoricesaponine $\mathrm{A} 3$ & GC \\
\hline 31 & 17.32 & 325.1427 & $269.0805\left[\mathrm{M}+\mathrm{H}-\mathrm{C}_{4} \mathrm{H}_{7}\right]^{+}$ & $\mathrm{C}_{20} \mathrm{H}_{20} \mathrm{O}_{4}$ & -2.355 & Bavachin & BGZ \\
\hline 32 & 18.00 & 455.2024 & $\begin{array}{l}440.1799\left[\mathrm{M}+\mathrm{Na}-\mathrm{CH}_{3}\right]^{+}, 409.1617\left[\mathrm{M}+\mathrm{H}-\mathrm{CH}_{3}-\right. \\
\left.\mathrm{OCH}_{3}\right]^{+}\end{array}$ & $\mathrm{C}_{24} \mathrm{H}_{32} \mathrm{O}_{7}$ & -3.525 & Schisandrol A & WWZ \\
\hline 33 & 18.14 & 323.1268 & $267.0646\left[\mathrm{M}+\mathrm{H}-\mathrm{C}_{4} \mathrm{H}_{8}\right]^{+}, 255.0652\left[\mathrm{M}+\mathrm{H}-\mathrm{C}_{5} \mathrm{H}_{8}\right]^{+}$ & $\mathrm{C}_{20} \mathrm{H}_{18} \mathrm{O}_{4}$ & -3.050 & Neobavaisoflavone & BGZ \\
\hline 35 & 18.77 & 249.1478 & $231.1379\left[\mathrm{M}+\mathrm{H}-\mathrm{H}_{2}\right]^{+}$ & $\mathrm{C}_{15} \mathrm{H}_{20} \mathrm{O}_{3}$ & -2.974 & Atractylenolide III & $\mathrm{BZ}$ \\
\hline 36 & 18.82 & 553.2030 & NA & $\mathrm{C}_{28} \mathrm{H}_{34} \mathrm{O}_{10}$ & -2.564 & Gomisin D & WWZ \\
\hline 37 & 18.82 & 231.1374 & $\begin{array}{l}213.1274[\mathrm{M}+\mathrm{H}-\mathrm{OH}]^{+}, 203.1067[\mathrm{M}+\mathrm{H}-\mathrm{CO}]^{+} \\
185.1325\left[\mathrm{M}+\mathrm{H}-\mathrm{CO}-\mathrm{H}_{2} \mathrm{O}\right]^{+}, 163.0753[\mathrm{M} \\
\left.+\mathrm{H}-\mathrm{C}_{5} \mathrm{H}_{8}\right]^{+}\end{array}$ & $\mathrm{C}_{15} \mathrm{H}_{18} \mathrm{O}_{2}$ & -2.537 & Atractylenolide I & BZ \\
\hline 38 & 18.82 & 325.1426 & $269.0805\left[\mathrm{M}+\mathrm{H}-\mathrm{C}_{4} \mathrm{H}_{7}\right]^{+}$ & $\mathrm{C}_{20} \mathrm{H}_{20} \mathrm{O}_{4}$ & -2.539 & $\begin{array}{l}\text { Bavachalcone/ } \\
\text { Isobavachalcone }\end{array}$ & BGZ \\
\hline 40 & 19.28 & 439.1711 & $\begin{array}{l}\text { 424.I485[M+Na-CH }]_{3}^{+}, 393.1303\left[\mathrm{M}+\mathrm{Na}-\mathrm{CH}_{3}-\right. \\
\left.\mathrm{OCH}_{3}\right]^{+}\end{array}$ & $\mathrm{C}_{23} \mathrm{H}_{28} \mathrm{O}_{7}$ & -3.744 & Schisandrol B & WWZ \\
\hline 43 & 20.57 & 389.2535 & $37 \mathrm{I} .2438\left[\mathrm{M}+\mathrm{H}-\mathrm{H}_{2} \mathrm{O}\right]^{+}$ & $\mathrm{C}_{20} \mathrm{H}_{36} \mathrm{O}_{7}$ & 0.257 & Tetradecylcitric acid & $\mathrm{BZ}$ \\
\hline 45 & 20.91 & 523.2281 & $\begin{array}{l}508.2060\left[\mathrm{M}+\mathrm{Na}-\mathrm{H}_{2} \mathrm{O}\right]^{+}, 477.1876[\mathrm{M}+\mathrm{Na}- \\
\left.\mathrm{C}_{2} \mathrm{H}_{6} \mathrm{O}\right]^{+}, 44 \mathrm{I} .1879\left[\mathrm{M}+\mathrm{Na}-\mathrm{C}_{5} \mathrm{H}_{6} \mathrm{O}\right]^{+}, 409.1617 \\
{\left[\mathrm{M}+\mathrm{Na}-\mathrm{C}_{6} \mathrm{H}_{10} \mathrm{O}_{2}\right]^{+}, 386.1719\left[\mathrm{M}+\mathrm{Na}-\mathrm{C}_{8} \mathrm{H}_{9} \mathrm{O}_{6}\right]^{+}}\end{array}$ & $\mathrm{C}_{28} \mathrm{H}_{36} \mathrm{O}_{8}$ & -4.108 & $\begin{array}{l}\text { Tigloylgomisin } \mathrm{H} / \\
\text { Angeloylgomisin } \mathrm{H}\end{array}$ & WWZ \\
\hline 46 & 21.21 & 523.2280 & $\begin{array}{l}508.2060\left[\mathrm{M}+\mathrm{Na}-\mathrm{H}_{2} \mathrm{O}\right]^{+}, 477.1876[\mathrm{M}+\mathrm{Na}- \\
\left.\mathrm{C}_{2} \mathrm{H}_{6} \mathrm{O}\right]^{+}, 441.1879\left[\mathrm{M}+\mathrm{Na}-\mathrm{C}_{5} \mathrm{H}_{6} \mathrm{O}\right]^{+}, 409.1617[\mathrm{M} \\
\left.+\mathrm{Na}-\mathrm{C}_{6} \mathrm{H}_{10} \mathrm{O}_{2}\right]^{+}, 386.1719\left[\mathrm{M}+\mathrm{Na}-\mathrm{C}_{8} \mathrm{H}_{9} \mathrm{O}_{6}\right]^{+}\end{array}$ & $\mathrm{C}_{28} \mathrm{H}_{36} \mathrm{O}_{8}$ & -4.337 & $\begin{array}{l}\text { Tigloylgomisin } \mathrm{H} / \\
\text { Angeloylgomisin } \mathrm{H}\end{array}$ & WWZ \\
\hline 48 & 21.67 & 233.1528 & $\begin{array}{l}215.1429\left[\mathrm{M}+\mathrm{H}-\mathrm{H}_{2} \mathrm{O}\right]^{+}, \mathrm{I} 87.1480[\mathrm{M} \\
\left.+\mathrm{H}-\mathrm{H}_{2} \mathrm{O}-\mathrm{CO}\right]^{+}, \mathrm{I} 1.0752\left[\mathrm{M}+\mathrm{H}-\mathrm{C}_{6} \mathrm{H}_{10}\right]^{+}\end{array}$ & $\mathrm{C}_{15} \mathrm{H}_{20} \mathrm{O}_{2}$ & -3.330 & Atractylenolide II & BZ \\
\hline 49 & 21.97 & 537.2079 & $\begin{array}{l}437.1566\left[\mathrm{M}+\mathrm{Na}-\mathrm{C}_{5} \mathrm{H}_{8} \mathrm{O}_{2}\right]^{+}, 415.175 \mathrm{I}[\mathrm{M}+\mathrm{Na}- \\
\left.\mathrm{C}_{7} \mathrm{H}_{6} \mathrm{O}_{2}\right]^{+}\end{array}$ & $\mathrm{C}_{28} \mathrm{H}_{34} \mathrm{O}_{9}$ & -3.004 & Tigloylgomisin P/Gomisin E & WWZ \\
\hline 50 & 22.74 & 339.1580 & $\begin{array}{l}32 \mathrm{I} .1483\left[\mathrm{M}+\mathrm{H}-\mathrm{H}_{2} \mathrm{O}\right]^{+}, 283.096 \mathrm{I}\left[\mathrm{M}+\mathrm{H}-\mathrm{C}_{4} \mathrm{H}_{8}\right]^{+}, \\
27 \mathrm{I} .0962\left[\mathrm{M}+\mathrm{H}-\mathrm{C}_{5} \mathrm{H}_{8}\right]^{+}\end{array}$ & $\mathrm{C}_{21} \mathrm{H}_{22} \mathrm{O}_{4}$ & -3.850 & Bavachinin A & BGZ \\
\hline 51 & 22.89 & 537.2073 & $\begin{array}{l}437.1566\left[\mathrm{M}+\mathrm{Na}-\mathrm{C}_{5} \mathrm{H}_{8} \mathrm{O}_{2}\right]^{+}, 415.175 \mathrm{I}[\mathrm{M}+\mathrm{Na}- \\
\left.\mathrm{C}_{7} \mathrm{H}_{6} \mathrm{O}_{2}\right]^{+}\end{array}$ & $\mathrm{C}_{28} \mathrm{H}_{34} \mathrm{O}_{9}$ & -4.139 & Tigloylgomisin P/Gomisin E & WWZ \\
\hline 53 & 25.31 & 339.1579 & $\begin{array}{l}32 \mathrm{I} .1482\left[\mathrm{M}+\mathrm{H}-\mathrm{H}_{2} \mathrm{O}\right]^{+}, 283.0962\left[\mathrm{M}+\mathrm{H}-\mathrm{C}_{4} \mathrm{H}_{8}\right]^{+}, \\
27 \mathrm{I} .0962\left[\mathrm{M}+\mathrm{H}-\mathrm{C}_{5} \mathrm{H}_{8}\right]^{+}\end{array}$ & $\mathrm{C}_{21} \mathrm{H}_{22} \mathrm{O}_{4}$ & -3.584 & 4'-O-methylbavachalcone & BGZ \\
\hline
\end{tabular}

Abbreviations: NA, not available.

\section{Statistical Analysis}

All experiments were performed in triplicate, and the data are presented as mean \pm standard deviation. Using GraphPad Prism version 7.0 (GraphPad Software, CA, USA) with Student's $t$-test, statistical analysis was conducted to evaluate the significance of differences between groups. Statistical significance was set at $P<0.05$.

\section{Results and Discussion}

\section{Identification of the Main Constituents of GBKC}

The chemical composition of GBKC was detected and identified by UHPLC-LTQ-Orbitrap (Figure S1 and S2). In total, 53 major components, including flavonoids and their glycosides, coumarins, lignans, steroids, alkaloids 
Table 2 Analysis of the Chemical Constituents of GBKC by UHPLC-LTQ-Orbitrap in Negative lon Mode

\begin{tabular}{|c|c|c|c|c|c|c|c|}
\hline No. & $\begin{array}{c}\text { RT } \\
\text { (Min) }\end{array}$ & $\begin{array}{c}{[\mathrm{M}-\mathrm{H}]^{-} /} \\
{\left[\mathrm{M}+\mathrm{HCOO}^{-}\right.}\end{array}$ & MS/MS & $\begin{array}{l}\text { Molecular } \\
\text { Formula }\end{array}$ & $\begin{array}{l}\text { Error } \\
\text { (ppm) }\end{array}$ & Compound & Herb \\
\hline I & 1.91 & 191.0190 & $173.0088\left[\mathrm{M}-\mathrm{H}-\mathrm{H}_{2} \mathrm{O}\right]^{-}, \mathrm{I} \mid \mathrm{I} .0088\left[\mathrm{M}-\mathrm{H}-\mathrm{CH}_{4} \mathrm{O}_{4}\right]^{-}$ & $\mathrm{C}_{6} \mathrm{H}_{8} \mathrm{O}_{7}$ & 1.995 & Citric acid/Isocitric acid & WWZ \\
\hline 2 & 2.26 & 191.0188 & $173.0088\left[\mathrm{M}-\mathrm{H}-\mathrm{H}_{2} \mathrm{O}\right]^{-}, \mathrm{I} / \mathrm{I} .0088\left[\mathrm{M}-\mathrm{H}-\mathrm{CH}_{4} \mathrm{O}_{4}\right]^{-}$ & $\mathrm{C}_{6} \mathrm{H}_{8} \mathrm{O}_{7}$ & 1.995 & Citric acid/lsocitric acid & WWZ \\
\hline 4 & 8.79 & 563.1379 & $\begin{array}{l}545.1290\left[\mathrm{M}-\mathrm{H}-\mathrm{H}_{2} \mathrm{O}\right]^{-}, 503.1184 \\
{\left[\mathrm{M}-\mathrm{H}-\mathrm{C}_{2} \mathrm{H}_{2} \mathrm{O}_{2}\right]^{-} 473.1080\left[\mathrm{M}-\mathrm{H}-\mathrm{C}_{3} \mathrm{H}_{6} \mathrm{O}_{3}\right]^{-},} \\
443.0974\left[\mathrm{M}-\mathrm{H}-\mathrm{C}_{4} \mathrm{H}_{8} \mathrm{O}_{4}\right]^{-}\end{array}$ & $\mathrm{C}_{26} \mathrm{H}_{28} \mathrm{O}_{14}$ & -1.652 & Schaftoside/lsoschaftoside & GC \\
\hline 5 & 9.01 & 365.0859 & $\begin{array}{l}203.0347\left[\mathrm{M}-\mathrm{H}-\mathrm{C}_{6} \mathrm{H}_{10} \mathrm{O}_{5}\right]^{-}, 159.0450 \\
{\left[\mathrm{M}-\mathrm{H}-\mathrm{C}_{6} \mathrm{H}_{10} \mathrm{O}_{5}-\mathrm{CO}_{2}\right]^{-}}\end{array}$ & $\mathrm{C}_{17} \mathrm{H}_{18} \mathrm{O}_{9}$ & -2.269 & $\begin{array}{l}\text { Psoralenoside/ } \\
\text { Isopsoralenoside }\end{array}$ & BGZ \\
\hline 6 & 9.26 & 365.0859 & $\begin{array}{l}203.0347\left[\mathrm{M}-\mathrm{H}-\mathrm{C}_{6} \mathrm{H}_{10} \mathrm{O}_{5}\right]^{-}, 159.0450 \\
{\left[\mathrm{M}-\mathrm{H}-\mathrm{C}_{6} \mathrm{H}_{10} \mathrm{O}_{5}-\mathrm{CO}_{2}\right]^{-}}\end{array}$ & $\mathrm{C}_{17} \mathrm{H}_{18} \mathrm{O}_{9}$ & -2.352 & $\begin{array}{l}\text { Psoralenoside/ } \\
\text { Isopsoralenoside }\end{array}$ & BGZ \\
\hline 7 & 9.54 & 563.1384 & $\begin{array}{l}545.1290\left[\mathrm{M}-\mathrm{H}-\mathrm{H}_{2} \mathrm{O}\right]^{-}, 503.1184 \\
{\left[\mathrm{M}-\mathrm{H}-\mathrm{C}_{2} \mathrm{H}_{2} \mathrm{O}_{2}\right]^{-}, 473.1080\left[\mathrm{M}-\mathrm{H}-\mathrm{C}_{3} \mathrm{H}_{6} \mathrm{O}_{3}\right]^{-},} \\
443.0974\left[\mathrm{M}-\mathrm{H}-\mathrm{C}_{4} \mathrm{H}_{8} \mathrm{O}_{4}\right]^{-}\end{array}$ & $\mathrm{C}_{26} \mathrm{H}_{28} \mathrm{O}_{14}$ & -1.102 & Schaftoside/Isoschaftoside & GC \\
\hline 8 & 10.30 & 417.1173 & $255.0656\left[\mathrm{M}-\mathrm{H}-\mathrm{C}_{6} \mathrm{H}_{10} \mathrm{O}_{5}\right]^{-}$ & $\mathrm{C}_{21} \mathrm{H}_{22} \mathrm{O}_{9}$ & -0.729 & Liquiritin & GC \\
\hline 9 & 10.32 & 255.0650 & I35.0088[M-H- $\left.\mathrm{C}_{8} \mathrm{H}_{8} \mathrm{O}\right]^{-}$ & $\mathrm{C}_{15} \mathrm{H}_{12} \mathrm{O}_{4}$ & -0.135 & Liquiritigenin & GC \\
\hline 10 & 10.39 & 549.1582 & $\begin{array}{l}417.1 \mathrm{I} 90\left[\mathrm{M}-\mathrm{H}-\mathrm{C}_{5} \mathrm{H}_{8} \mathrm{O}_{4}\right]^{-}, 297.0764 \\
{\left[\mathrm{M}-\mathrm{H}-\mathrm{C}_{5} \mathrm{H}_{8} \mathrm{O}_{4}-\mathrm{C}_{4} \mathrm{H}_{6} \mathrm{O}_{4}\right]^{-}, 255.066 \mathrm{I}} \\
{\left[\mathrm{M}-\mathrm{H}-\mathrm{C}_{5} \mathrm{H}_{8} \mathrm{O}_{4}-\mathrm{C}_{4} \mathrm{H}_{6} \mathrm{O}_{4}-\mathrm{C}_{2} \mathrm{H}_{2} \mathrm{O}\right]^{-}}\end{array}$ & $\mathrm{C}_{26} \mathrm{H}_{30} \mathrm{O}_{13}$ & -2.067 & Liquiritin apioside & GC \\
\hline 11 & 10.42 & 255.0650 & $135.0088\left[\mathrm{M}-\mathrm{H}-\mathrm{C}_{8} \mathrm{H}_{8} \mathrm{O}\right]^{-}$ & $\mathrm{C}_{15} \mathrm{H}_{12} \mathrm{O}_{4}$ & -0.844 & Isoliquiritigenin & GC \\
\hline 12 & 10.42 & 425.2007 & $263.1493\left[\mathrm{M}-\mathrm{H}-\mathrm{C}_{6} \mathrm{H}_{10} \mathrm{O}_{5}\right]^{-}, 161.0453\left[\mathrm{C}_{6} \mathrm{H}_{9} \mathrm{O}_{5}\right]^{-}$ & $\mathrm{C}_{18} \mathrm{H}_{34} \mathrm{O}_{11}$ & -2.559 & Hexyl- $\beta$-D-maltoside & DS \\
\hline 13 & 10.89 & 395.1903 & $263.1493\left[\mathrm{M}-\mathrm{H}-\mathrm{C}_{5} \mathrm{H}_{8} \mathrm{O}_{4}\right]^{-}, 161.0453\left[\mathrm{C}_{6} \mathrm{H}_{9} \mathrm{O}_{5}\right]^{-}$ & $\mathrm{C}_{17} \mathrm{H}_{32} \mathrm{O}_{10}$ & -0.834 & Dextran & DS \\
\hline 14 & 12.11 & 549.1586 & $\begin{array}{l}417.1 \mathrm{I} 90\left[\mathrm{M}-\mathrm{H}-\mathrm{C}_{5} \mathrm{H}_{8} \mathrm{O}_{4}\right]^{-}, 297.0764 \\
{\left[\mathrm{M}-\mathrm{H}-\mathrm{C}_{5} \mathrm{H}_{8} \mathrm{O}_{4}-\mathrm{C}_{4} \mathrm{H}_{6} \mathrm{O}_{4}\right]^{-}, 255.066 \mathrm{I}} \\
{\left[\mathrm{M}-\mathrm{H}-\mathrm{C}_{5} \mathrm{H}_{8} \mathrm{O}_{4}-\mathrm{C}_{4} \mathrm{H}_{6} \mathrm{O}_{4}-\mathrm{C}_{2} \mathrm{H}_{2} \mathrm{O}\right]^{-}}\end{array}$ & $\mathrm{C}_{26} \mathrm{H}_{30} \mathrm{O}_{13}$ & -1.637 & Liquiritin apioside isomer & GC \\
\hline 15 & 12.44 & 417.1173 & $255.0656\left[\mathrm{M}-\mathrm{H}-\mathrm{C}_{6} \mathrm{H}_{10} \mathrm{O}_{5}\right]^{-}$ & $\mathrm{C}_{21} \mathrm{H}_{22} \mathrm{O}_{9}$ & -0.729 & Isoliquiritin & GC \\
\hline 16 & 12.59 & 475.1227 & NA & $\mathrm{C}_{22} \mathrm{H}_{22} \mathrm{O}_{9}$ & -1.764 & Ononin & GC \\
\hline 17 & 12.59 & 267.0649 & NA & $\mathrm{C}_{16} \mathrm{H}_{12} \mathrm{O}_{4}$ & -3.084 & Formononetin & GC \\
\hline 22 & 14.77 & 837.3875 & $\begin{array}{l}819.3779\left[\mathrm{M}-\mathrm{H}-\mathrm{H}_{2} \mathrm{O}\right]^{-}, 661.3566 \\
{\left[\mathrm{M}-\mathrm{H}-\mathrm{C}_{6} \mathrm{H}_{8} \mathrm{O}_{6}\right]^{-}, 35 \mathrm{I} .0552\left[\mathrm{M}-\mathrm{H}-\mathrm{C}_{30} \mathrm{H}_{46} \mathrm{O}_{4}\right]^{-}}\end{array}$ & $\mathrm{C}_{42} \mathrm{H}_{62} \mathrm{O}_{17}$ & -4.090 & Licoricesaponin G2/isomer & GC \\
\hline 23 & 15.58 & 837.3909 & $\begin{array}{l}819.377 \mathrm{I}\left[\mathrm{M}-\mathrm{H}-\mathrm{H}_{2} \mathrm{O}\right]^{-}, 661.3569 \\
{\left[\mathrm{M}-\mathrm{H}-\mathrm{C}_{6} \mathrm{H}_{8} \mathrm{O}_{6}\right]^{-}, 35 \mathrm{I} .0553\left[\mathrm{M}-\mathrm{H}-\mathrm{C}_{30} \mathrm{H}_{46} \mathrm{O}_{5}\right]^{-}}\end{array}$ & $\mathrm{C}_{42} \mathrm{H}_{62} \mathrm{O}_{17}$ & 0.649 & Licoricesaponin G2/isomer & GC \\
\hline 24 & 15.58 & 329.2321 & $\begin{array}{l}31 \mathrm{I} .2220\left[\mathrm{M}-\mathrm{H}-\mathrm{H}_{2} \mathrm{O}\right]^{-}, 293.2117\left[\mathrm{M}-\mathrm{H}-2 \mathrm{H}_{2} \mathrm{O}\right]^{-} \text {, } \\
229.1438\left[\mathrm{M}-\mathrm{H}-\mathrm{C}_{6} \mathrm{H}_{12} \mathrm{O}\right]^{-}, 21 \mathrm{I} .1335 \\
{\left[\mathrm{M}-\mathrm{H}-\mathrm{C}_{6} \mathrm{H}_{12} \mathrm{O}_{2}\right]^{-}, 17 \mathrm{I} .1024\left[\mathrm{C}_{9} \mathrm{H}_{15} \mathrm{O}_{3}\right]^{-}}\end{array}$ & $\mathrm{C}_{18} \mathrm{H}_{34} \mathrm{O}_{5}$ & -0.488 & $\begin{array}{l}\text { 9(S), I2(S), 13(S)-trihydroxy-10 } \\
\text { (E)-octadecenoic Acid }\end{array}$ & DS \\
\hline 26 & 16.42 & 821.3919 & $\begin{array}{l}803.382 \mathrm{I}\left[\mathrm{M}-\mathrm{H}-\mathrm{H}_{2} \mathrm{O}\right]^{-}, 759.3926 \\
{\left[\mathrm{M}-\mathrm{H}-\mathrm{H}_{2} \mathrm{O}-\mathrm{CO}_{2}\right]^{-}, 645.36 \mathrm{II}\left[\mathrm{M}-\mathrm{H}-\mathrm{C}_{6} \mathrm{H}_{8} \mathrm{O}_{6}\right]^{-},} \\
627.3506\left[\mathrm{M}-\mathrm{H}-\mathrm{C}_{6} \mathrm{H}_{10} \mathrm{O}_{7}\right]^{-}, 35 \mathrm{I} .0548 \\
{\left[\mathrm{C}_{12} \mathrm{H}_{15} \mathrm{O}_{12}\right]^{-}}\end{array}$ & $\mathrm{C}_{42} \mathrm{H}_{62} \mathrm{O}_{16}$ & -4.931 & Glycyrrhizic acid & GC \\
\hline 27 & 16.44 & 327.2167 & $\begin{array}{l}309.2063\left[\mathrm{M}-\mathrm{H}-\mathrm{H}_{2} \mathrm{O}\right]^{-}, 291.1958\left[\mathrm{M}-\mathrm{H}-2 \mathrm{H}_{2} \mathrm{O}\right]^{-} \\
229.1438\left[\mathrm{M}-\mathrm{H}-\mathrm{C}_{6} \mathrm{H}_{10} \mathrm{O}_{7}\right]^{-}, 211.1334 \\
{\left[\mathrm{M}-\mathrm{H}-\mathrm{C}_{6} \mathrm{H}_{10} \mathrm{O}_{7}-\mathrm{H}_{2} \mathrm{O}\right]^{-}, 17 \mathrm{I} .1023\left[\mathrm{C}_{9} \mathrm{H}_{15} \mathrm{O}_{3}\right]^{-}}\end{array}$ & $\mathrm{C}_{19} \mathrm{H}_{20} \mathrm{O}_{5}$ & 0.335 & Methylophiopogonanone B & MD \\
\hline
\end{tabular}


Table 2 (Continued).

\begin{tabular}{|c|c|c|c|c|c|c|c|}
\hline No. & $\begin{array}{c}\text { RT } \\
\text { (Min) }\end{array}$ & $\begin{array}{c}{[\mathrm{M}-\mathrm{H}]^{-} /} \\
{[\mathrm{M}+\mathrm{HCOO}]^{-}}\end{array}$ & MS/MS & $\begin{array}{l}\text { Molecular } \\
\text { Formula }\end{array}$ & $\begin{array}{l}\text { Error } \\
(\mathrm{ppm})\end{array}$ & Compound & Herb \\
\hline 29 & 17.27 & 821.3919 & $\begin{array}{l}803.3820\left[\mathrm{M}-\mathrm{H}-\mathrm{H}_{2} \mathrm{O}\right]^{-}, 645.36 \mathrm{II} \\
{\left[\mathrm{M}-\mathrm{H}-\mathrm{C}_{6} \mathrm{H}_{8} \mathrm{O}_{6}\right]^{-}}\end{array}$ & $\mathrm{C}_{42} \mathrm{H}_{62} \mathrm{O}_{16}$ & -4.931 & Uralsaponin B & GC \\
\hline 30 & 17.30 & 983.4451 & $\begin{array}{l}965.4338\left[\mathrm{M}-\mathrm{H}-\mathrm{H}_{2} \mathrm{O}\right]^{-}, 893.4147 \\
{\left[\mathrm{M}-\mathrm{H}-\mathrm{C}_{3} \mathrm{H}_{7} \mathrm{O}_{3}\right]^{-}, 82 \mathrm{I} .3914\left[\mathrm{M}-\mathrm{H}-\mathrm{C}_{6} \mathrm{H}_{10} \mathrm{O}_{5}\right]^{-},} \\
803.3823\left[\mathrm{M}-\mathrm{H}-\mathrm{C}_{6} \mathrm{H}_{10} \mathrm{O}_{5}-\mathrm{H}_{2} \mathrm{O}\right]^{-}\end{array}$ & $\mathrm{C}_{48} \mathrm{H}_{72} \mathrm{O}_{21}$ & -3.776 & Licoricesaponine $\mathrm{A} 3$ & GC \\
\hline 31 & 17.32 & 323.1275 & $203.0709\left[\mathrm{M}-\mathrm{H}-\mathrm{C}_{8} \mathrm{H}_{8} \mathrm{O}\right]^{-}$ & $\mathrm{C}_{20} \mathrm{H}_{20} \mathrm{O}_{4}$ & -0.977 & Bavachin & BGZ \\
\hline 33 & 18.14 & 321.1117 & $265.0504\left[\mathrm{M}-\mathrm{H}-\mathrm{C}_{4} \mathrm{H}_{8}\right]^{-}$ & $\mathrm{C}_{20} \mathrm{H}_{18} \mathrm{O}_{4}$ & -1.481 & Neobavaisoflavone & BGZ \\
\hline 34 & 18.64 & 359.1124 & $344.0891\left[\mathrm{M}-\mathrm{H}-\mathrm{CH}_{3}\right]^{-}$ & $\mathrm{C}_{19} \mathrm{H}_{20} \mathrm{O}_{7}$ & -0.159 & $\begin{array}{l}\text { 5,7-dihydroxy-3-(2-hydroxy- } \\
\text { 4-methoxybenzyl)- } \\
\text { 8-methoxy-6-methylchroman- } \\
\text { 4-one }\end{array}$ & MD \\
\hline 38 & 18.82 & 323.1269 & $203.0709\left[\mathrm{M}-\mathrm{H}-\mathrm{C}_{8} \mathrm{H}_{8} \mathrm{O}\right]^{-}$ & $\mathrm{C}_{20} \mathrm{H}_{20} \mathrm{O}_{4}$ & -2.679 & $\begin{array}{l}\text { Bavachalcone/ } \\
\text { Isobavachalcone }\end{array}$ & BGZ \\
\hline 39 & 18.94 & 783.4 & NA & $\mathrm{C}_{39} \mathrm{H}_{62} \mathrm{O}_{13}$ & -0.252 & Dracaenoside $F$ & MD \\
\hline 41 & 19.89 & 319.0965 & $304.0742\left[\mathrm{M}-\mathrm{H}-\mathrm{CH}_{3}\right]^{-}, 30 \mathrm{I} .0863\left[\mathrm{M}-\mathrm{H}-\mathrm{H}_{2} \mathrm{O}\right]^{-}$ & $\mathrm{C}_{20} \mathrm{H}_{16} \mathrm{O}_{4}$ & 0.046 & Corylin & BGZ \\
\hline 42 & 20.48 & 335.0913 & $280.037 \mathrm{I}\left[\mathrm{M}-\mathrm{H}-\mathrm{C}_{4} \mathrm{H}_{7}\right]^{-}$ & $\mathrm{C}_{20} \mathrm{H}_{16} \mathrm{O}_{5}$ & -0.179 & Derrone/lsoderrone & GC \\
\hline 44 & 20.88 & 313.2374 & $\begin{array}{l}295.227 \mathrm{I}\left[\mathrm{M}-\mathrm{H}-\mathrm{H}_{2} \mathrm{O}\right]^{-}, 277.2166\left[\mathrm{M}-\mathrm{H}-2 \mathrm{H}_{2} \mathrm{O}\right]^{-} \\
201.1128\left[\mathrm{M}-\mathrm{H}-\mathrm{C}_{8} \mathrm{H}_{16}\right], \text { I } 17.1024\left[\mathrm{C}_{9} \mathrm{H}_{15} \mathrm{O}_{3}\right]^{-}\end{array}$ & $\mathrm{C}_{18} \mathrm{H}_{34} \mathrm{O}_{4}$ & 0.109 & $\begin{array}{l}9,10 \text {-dihydroxy-12- } \\
\text { octadecenoic acid }\end{array}$ & DS \\
\hline 47 & 21.58 & 351.0864 & $\begin{array}{l}283.0969\left[\mathrm{M}-\mathrm{H}-\mathrm{CH}_{3} \mathrm{O}\right]^{-}, 307.0967 \\
{\left[\mathrm{M}-\mathrm{H}-\mathrm{CO}_{2}\right]^{-}, 265.0862\left[\mathrm{M}-\mathrm{H}-\mathrm{C}_{3} \mathrm{H}_{2} \mathrm{O}_{3}\right]^{-}}\end{array}$ & $\mathrm{C}_{20} \mathrm{H}_{16} \mathrm{O}_{6}$ & -1.319 & I-methoxyphaseollidin & GC \\
\hline 52 & 23.49 & 389.1749 & $\begin{array}{l}277.0499\left[\mathrm{M}-\mathrm{H}-\mathrm{C}_{8} \mathrm{H}_{6}\right]^{-}, 265.0500 \\
{\left[\mathrm{M}-\mathrm{H}-\mathrm{C}_{9} \mathrm{H}_{6}\right]^{-}}\end{array}$ & $\mathrm{C}_{25} \mathrm{H}_{26} \mathrm{O}_{4}$ & 0.345 & Corylifol A & BGZ \\
\hline
\end{tabular}

Abbreviation: NA, not available.

and phenolic acids, were confirmed or tentatively identified based on comparison with the published data, according to retention times, molecular ions, fragmentation data, and fragmentation rules. As depicted in Figure S3-S12, liquiritigenin (peak 9), codonopyrrolidium A (peak18), psoralen (peak 19), glycyrrhizic acid (peak 26) and schisandrol A (peak 32) were chosen as examples to illustrate the mass fragmentation patterns of the components with the same basic skeletons. The detailed information of the identified compounds was summarized in Tables 1 and 2 .

\section{Putative Targets for GBKC}

As given in Table S1, 594 GBKC-related targets were predicted by MedChem Studio.

\section{CB-Related Targets}

After eliminating the repetitive targets, 414 predictive targets associated with CB were reserved. Among them, 138 targets were from DrugBank database and 286 genes were from OMIM database. The details were shown in Table S2.

\section{Network and Pathway Analysis}

To achieve a systematic understanding of the complex relationships, a "compound-target-disease" network containing the potential active components, GBKC-related targets, and CB-related targets was established based on the PPI information in the STRING database. There were 851 nodes and 12,548 edges in the network (Table S3).

The degree value of each node in the network was calculated to identify the central nodes. Ultimately, 423 nodes with an average degree value of $>23$ were identified as hubs. The interaction network was then established based on direct connections among the hubs. After conditional screening with topological coefficients ("degree" > 33, "betweenness" > 0.0011, and 
A

GO: BP

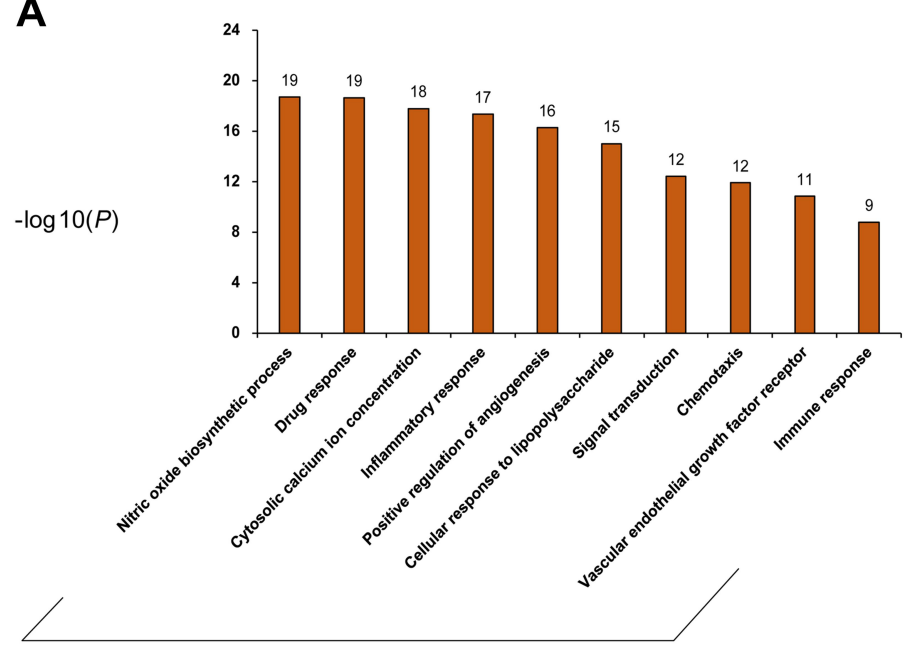

B

GO: CC

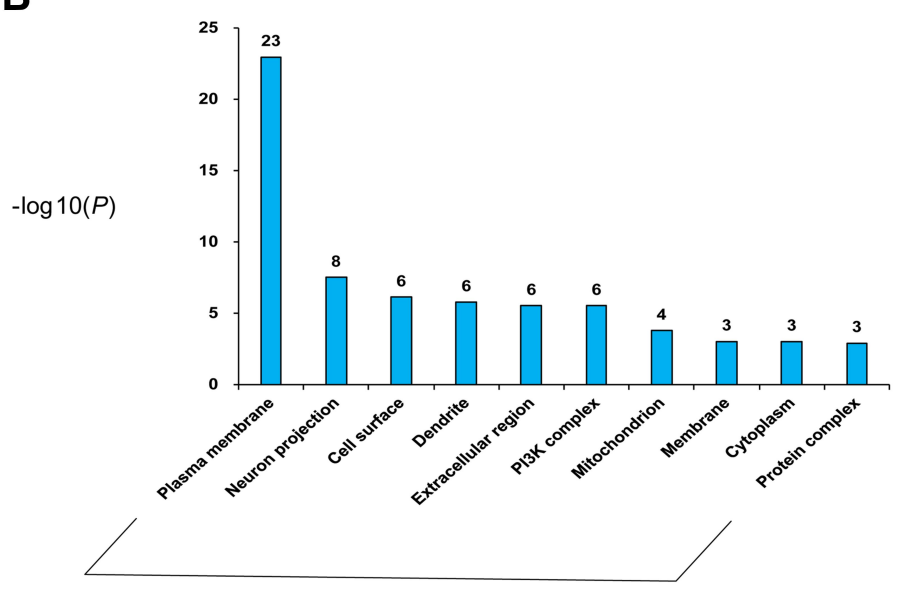

C

GO: MF

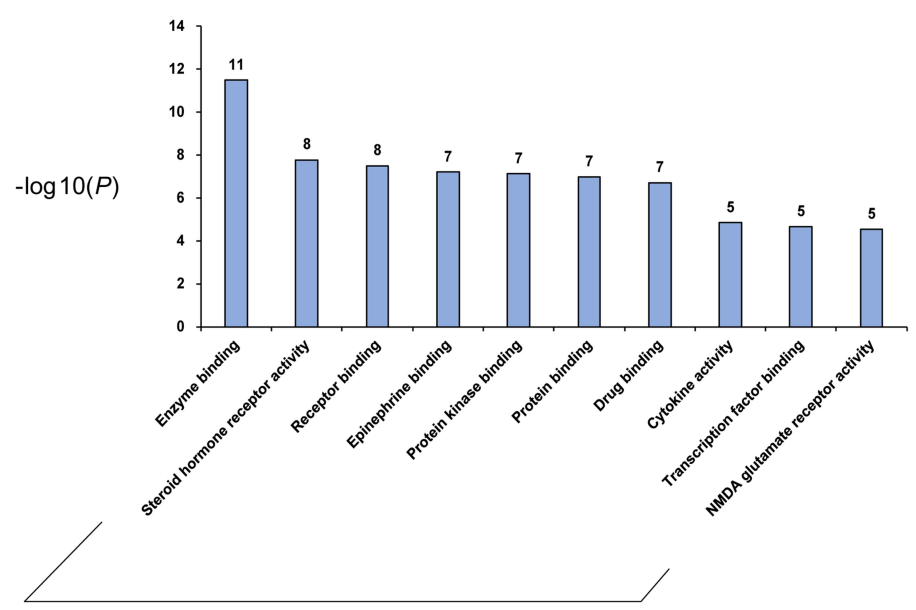

Figure 2 GO term performance of the major hubs. (A) GO: BP; (B) GO: CC; (C) GO: MF. The abscissa stands for GO terms, the ordinate stands for minus log 10(P). 


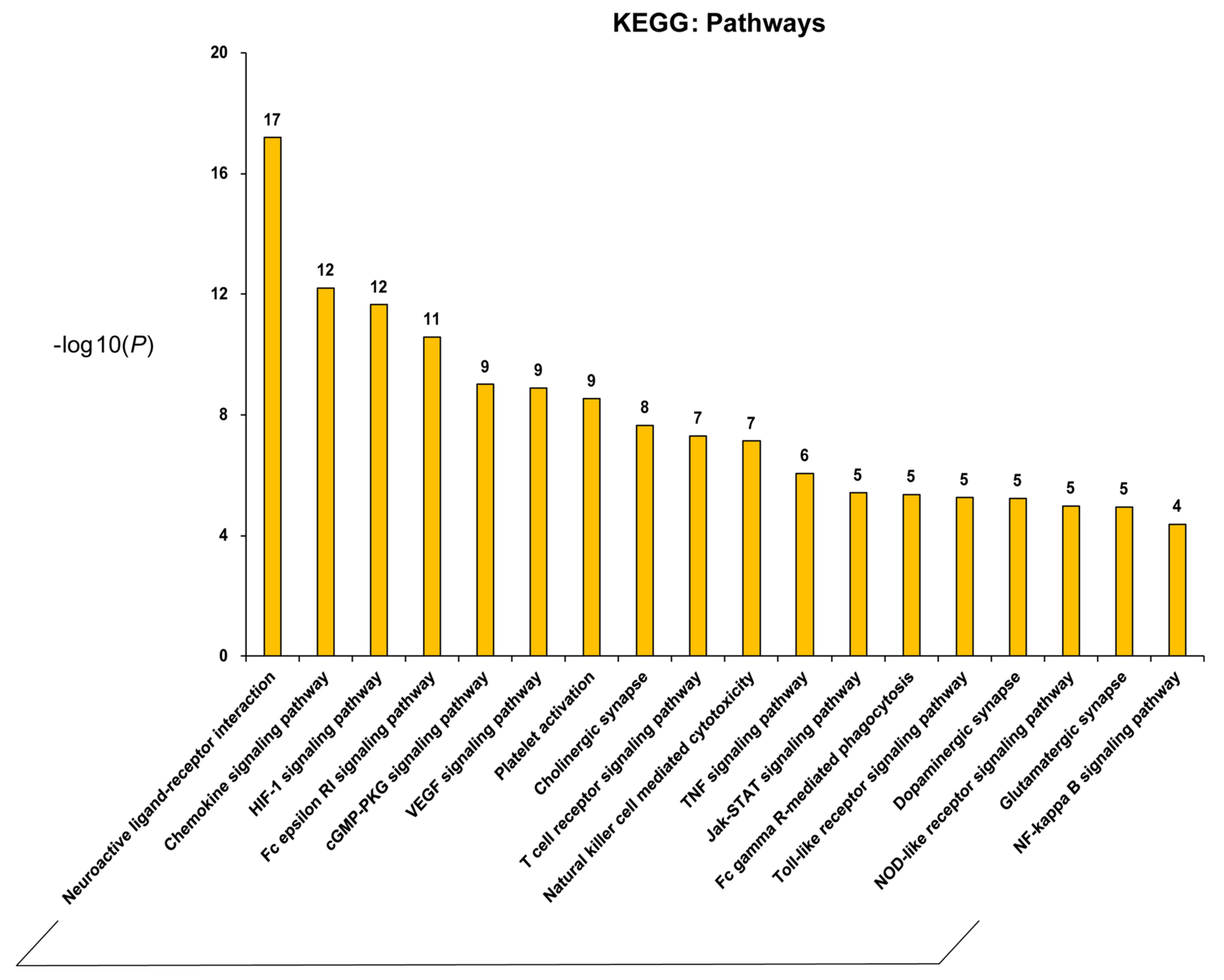

Figure 3 Main KEGG pathways composed of the major hubs. The abscissa stands for KEGG pathways, the ordinate stands for minus log $10(P)$.

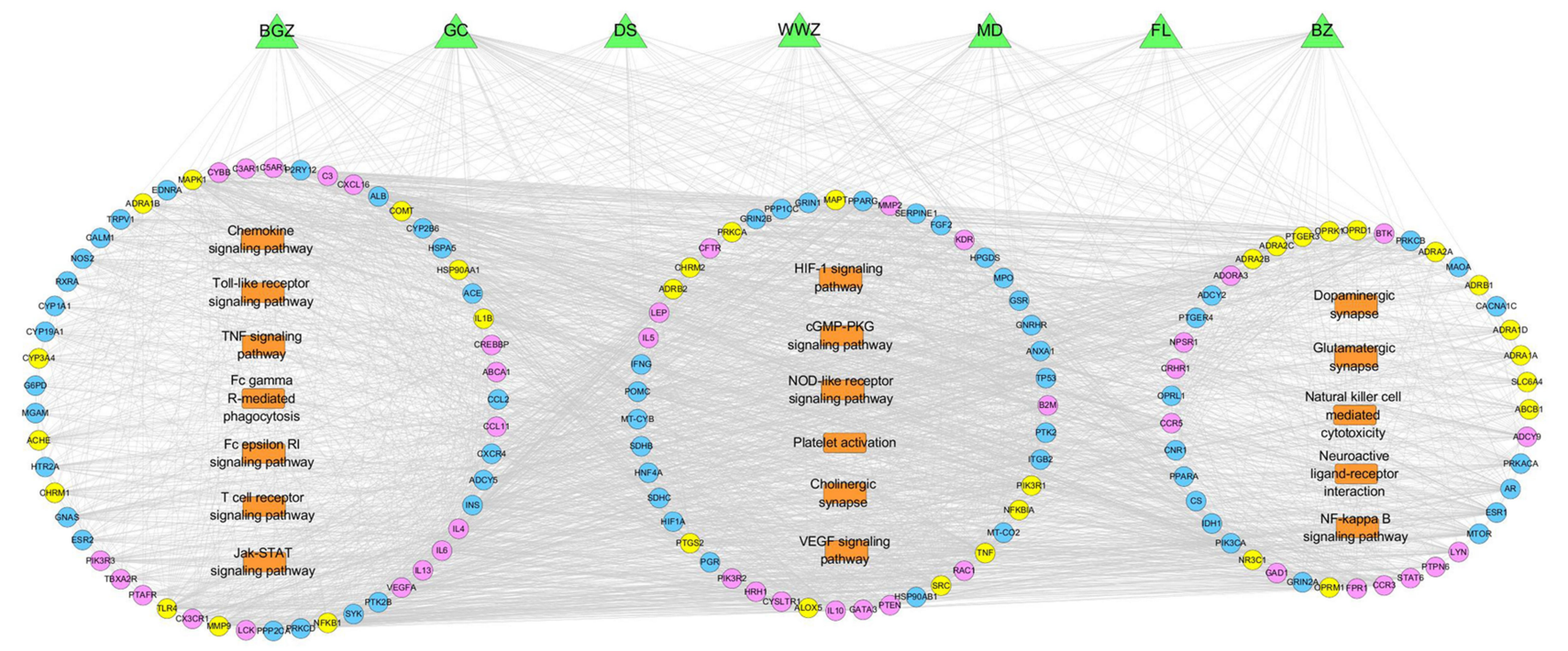

Figure 4 GBKC-major hubs-main pathway network. Green triangles represent each herbs contained in GBKC; round blue nodes represent putative targets of GBKC; round red nodes represent $C B$ associated targets; round yellow nodes represent both $G B K C$ targets and $C B$ associated targets; orange rectangles represent top 18 pathways from enrichment analysis of major targets; edges represent interactions among herbs in GBKC, putative targets, CB associated targets, and main pathways. 


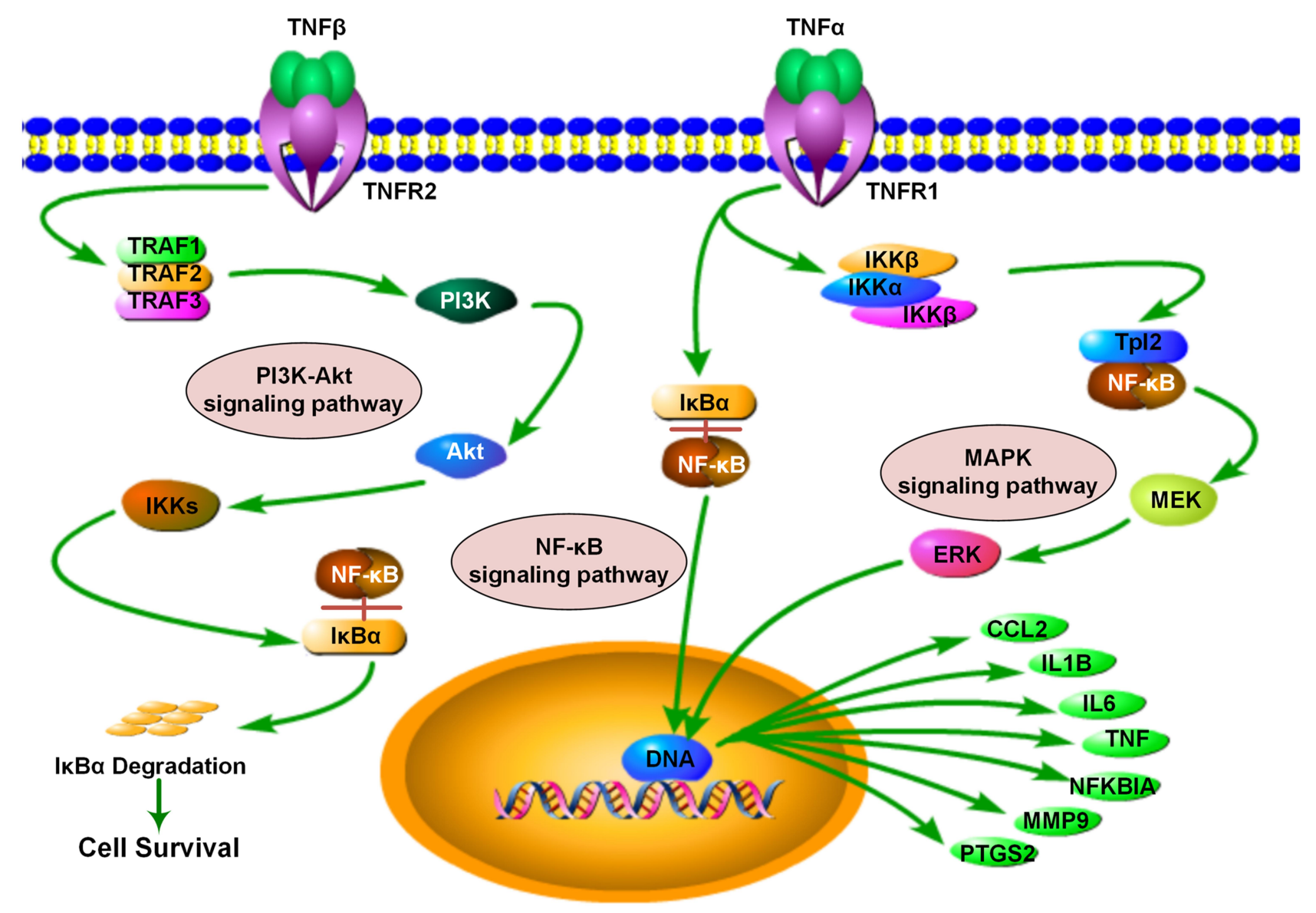

Figure 5 Illustration of TNF signaling pathway influenced by major putative targets of GBKC.

"closeness" > 0.4626), 141 hubs were considered as pivotal targets. Among them, 65 hubs were relevant to GBKC, 42 were relevant to $\mathrm{CB}$, and 34 were both GBKC and CB-associated targets. The details are presented in Table S4.

To clarify the pathways involved in the major hubs, GO enrichment analysis was conducted, which was composed of biological process (BP), cell component (CC), and molecular function (MF). As described in Figure $2 \mathrm{~A}-\mathrm{C}$, the top 10 significant terms of $\mathrm{BP}, \mathrm{CC}$, and $\mathrm{MF}$ were obtained. GBKC mainly participates in regulating the following BPs: nitric oxide biosynthetic process, drug response, cytosolic calcium ion concentration, inflammatory response, positive regulation of angiogenesis, cellular response to lipopolysaccharide, signal transduction, chemotaxis, vascular endothelial growth factor receptor, and immune response. GBKC affected $\mathrm{CB}$ by regulating the following MFs: enzyme binding, steroid hormone receptor activity, receptor binding, epinephrine binding, protein kinase binding, protein binding, drug binding, cytokine activity, transcription factor binding, and NMDA glutamate receptor activity. For $\mathrm{CCs}$, the major hubs were enriched in the plasma membrane, neuron projection, cell surface, dendrite, extracellular region, PI3K complex, mitochondrion, membrane, cytoplasm, and protein complex.

To illustrate the signaling pathways that have remarkably changed in the course of $\mathrm{CB}$, KEGG pathway enrichment analysis was performed. A total of 134 significant pathways were identified, and the top 18 signal pathways were depicted in Figure 3. These pathways were divided into the following three functional modules: immune system (such as Toll-like receptor signaling pathway, JAK-STAT signaling pathway, $T$ cell receptor signaling pathway, Fc epsilon RI signaling pathway, Fc gamma R-mediated phagocytosis, chemokine signaling pathway, and TNF signaling pathway), blood circulation system (such as NOD-like receptor signaling pathway, cholinergic synapse, cGMP-PKG 
signaling pathway, platelet activation, VEGF signaling pathway, and HIF-1 signaling pathway), and nervous system (such as neuroactive ligand-receptor interaction, dopaminergic synapse, NF-kappa B signaling pathway, natural killer cell mediated cytotoxicity, and glutamatergic synapse).

To facilitate a clear interpretation of the complex connections among GBKC components, key hubs of GBKC, and meaningful pathways, the compound-target-pathway network was graphed, as shown in Figure 4. Interestingly, the TNF signaling pathway was highly enriched in the main KEGG pathways. Previous studies have shown that TNF- $\alpha$ levels increase markedly in peripheral blood, bronchoalveolar lavage fluid, bronchial biopsies, and induced sputum of patients with $\mathrm{CB}^{28,29}$ Therefore, GBKC may ameliorate airway inflammation in patients with CB.

However, the network pharmacology method used in this study had some limitations. First, the components in GBKC were treated equally, without considering their contents. Second, some identified constituents may not be absorbed into the blood circulatory system, and in many situations, it is the metabolites, not the parent compounds, which have therapeutic effects. Third, the GBKC decoction is not suitable for CB with all TCM syndromes. Therefore, using targets related to the specific syndrome of $\mathrm{CB}$ to construct a network model would be more accurate.

\section{GBKC Attenuates the Inflammatory Response of CB Patients by Regulating the TNF Signaling Pathway}

As shown in Table S5, the GBKC-related targets associated with TNF signaling pathway include TNF- $\alpha$,

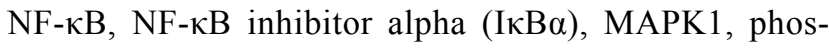
phatidylinositol-4,5-bisphosphate 3-kinase catalytic subunit alpha isoform (PIK3CA), phosphoinositide3-kinase regulatory subunit 1 (PIK3R1), PIK3R2, PIK3R3, MMP-9, prostaglandin-endoperoxide synthase 2 (PTGS2), IL-1 $\beta$, IL-6, and C-C motif chemokine 2 (CCL2). Figure 5 shows a graphical overview of the TNF pathway influenced by the major putative targets of GBKC.

\section{Molecular Docking}

Table 3 depicts the docking scores (cut-off value of 80) of GBKC hit components against the active sites of the
Table 3 Molecular Docking Results

\begin{tabular}{|l|c|c|}
\hline Targets & Compound & LibDockscore \\
\hline MMP9 & Liquiritin & 155.244 \\
MMP9 & Isoliquiritin & 125.357 \\
TNF & Isoliquiritin & 120.423 \\
MAPKI & Ononin & 120.082 \\
NFKBIA & Isoliquiritigenin & 117.096 \\
MAPKI & Liquiritin & 112.194 \\
PIK3CA & I-Methoxyphaseollidin & 109.393 \\
NFKBI & Isoliquiritigenin & 109.047 \\
MAPKI & Isopsoralenoside & 107.812 \\
MAPKI & Psoralenoside & 107.07 \\
MAPKI & Bavachin & 105.343 \\
PIK3CA & Corylifol A & 104.358 \\
MAPKI & Bavachinin A & 103.145 \\
PTGS2 & 9,10-Dihydroxy-I2-octadecenoic acid & 101.172 \\
NFKBI & Formononetin & 100.175 \\
MAPKI & Isoliquiritin & 99.9182 \\
NFKBIA & Formononetin & 95.6731 \\
MAPKI & 5,7-Dihydroxy-3-(2-hydroxy- & 92.7798 \\
& 4-methoxybenzyl)-8-methoxy- & \\
& 6-methylchroman-4-one & \\
MAPKI & Neobavaisoflavone & 88.7266 \\
MAPKI & 5,7-Dihydroxy-3-(2-hydroxy- & 83.3577 \\
& 4-methoxybenzyl)-8-methoxy- & \\
& 6-methylchroman-4-one & \\
\hline
\end{tabular}

identified protein targets in the TNF pathway, including MMP-9, TNF- $\alpha$, MAPK1, NFKBIA, NFKB1, and PTGS2. The major binding interactions between the above active compounds and target proteins include hydrogen bonding and amide-pi stacked, pi-pi stacked, pi-alkyl, and alkyl interactions. Herein, the molecular simulation of isoliquiritin in the active site of TNF- $\alpha$ was selected as an example to demonstrate how the active compounds bound to their targets (Figure 6). Isoliquiritin forms six hydrogen bonds, two of them were phenolic hydroxyl with LEU 157 and LEU 55. The other four, namely hydroxyl groups on glucose were with LEU 126, GLN 125 and SER 95. GLU 53 and GLY 54 were bound with other interactions, including amide-pi stacked and pi-sigma interactions. The molecular docking results described above are helpful for a basic understanding of the mechanism of drug action.

\section{SPR Assays for Affinity}

SPR assay was used to confirm the direct binding efficacy of the targets and their corresponding ligands based on the molecular docking results, including isoliquiritigenin-NFKB1, formononetin-NFKB1, liquiritin-MMP-9, isoliquiritin-MMP-9, liquiritin-TNF- $\alpha, \quad$ isoliquiritin-TNF- $\alpha$, ononin-MAPK1, 


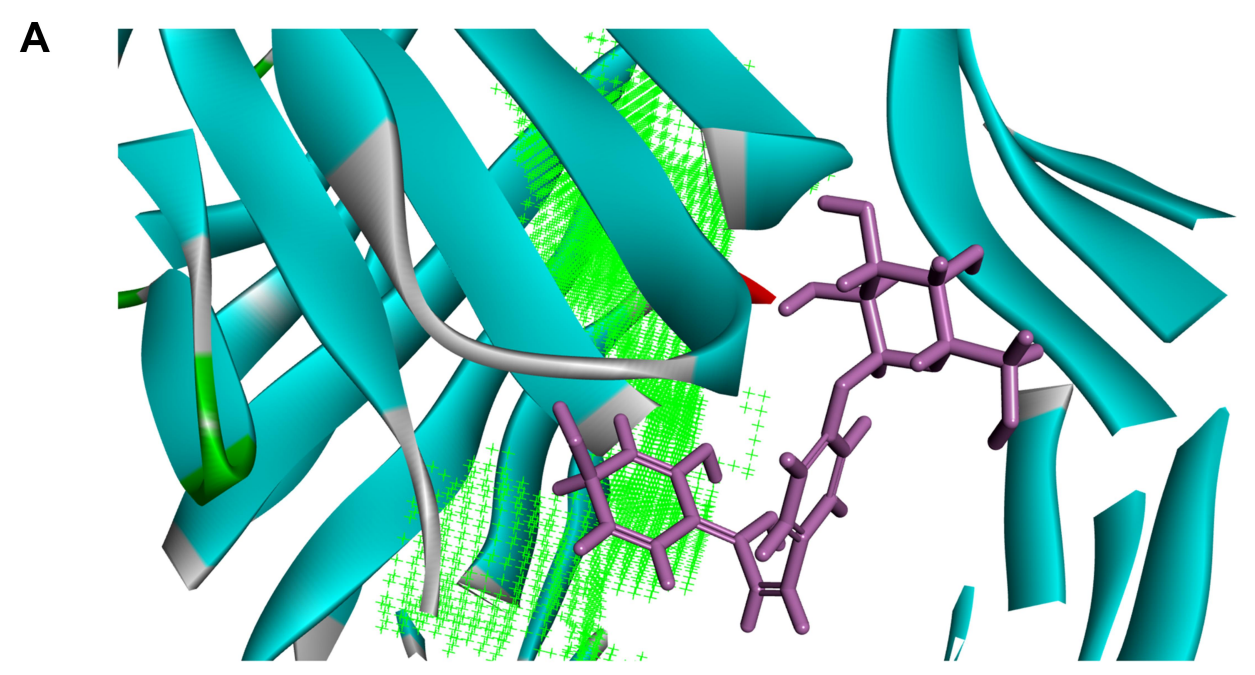

B

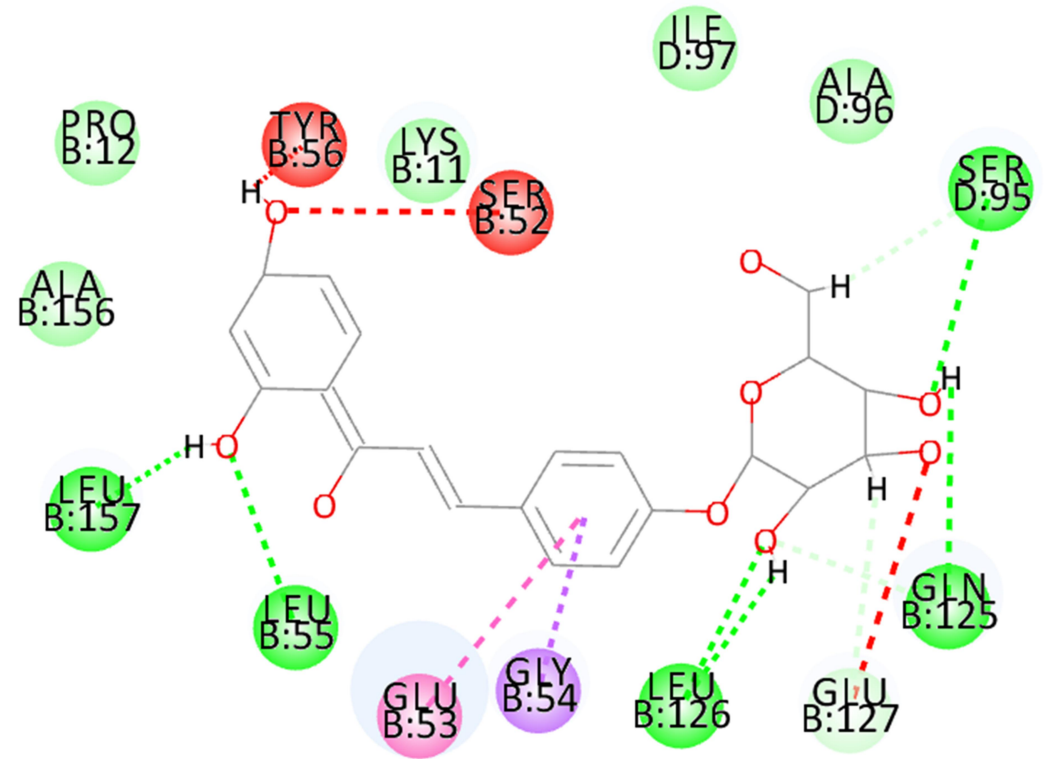

\section{Interactions}

van der Waals

Conventional Hydrogen Bond

Carbon Hydrogen Bond

Unfavorable Donor-Donor
Unfavorable Acceptor-Acceptor

Pi-Sigma

Amide-Pi Stacked

Figure 6 The binding modes of Isoliquiritin and protein TNF- $\alpha$. (A) Schematic (3D) representation and (B) Schematic (2D) representation of the interplay between isoliquiritin and TNF- $\alpha$ (PDB IDchimeric 2AZ5). The compounds were shown as stick model with purple colored and the others were active site amino acid residues.

liquiritin-MAPK1, isopsoralenoside-MAPK1, psoralenosideMAPK1, bavachin-MAPK1, bavachinin A-MAPK1, and isoliquiritin-MAPK1. As shown in Figure 7, we have found some ideal molecule-protein pairs that have relatively better affinities, including formononetin-NFKB1 $\left(\mathrm{K}_{\mathrm{D}}=32.5 \mu \mathrm{M}\right)$, isoliquiritin-TNF- $\alpha\left(\mathrm{K}_{\mathrm{D}}=3.6 \mu \mathrm{M}\right)$, isoliquiritin-MMP-9 $\left(\mathrm{K}_{\mathrm{D}}=\right.$ $2.7 \mu \mathrm{M})$, liquiritin-MMP-9 $\left(\mathrm{K}_{\mathrm{D}}=105 \mu \mathrm{M}\right)$, bavachin-MAPK1
$\left(\mathrm{K}_{\mathrm{D}}=26.6 \mu \mathrm{M}\right)$, bavachinin A-MAPK1 $\left(\mathrm{K}_{\mathrm{D}}=6.05 \mu \mathrm{M}\right)$, ononin-MAPK1 $\left(\mathrm{K}_{\mathrm{D}}=41.4 \mu \mathrm{M}\right)$, and liquiritin-MAPK1 $\left(\mathrm{K}_{\mathrm{D}}=382 \mu \mathrm{M}\right)$. It was interesting to note that liquiritinMMP-9 $\left(\mathrm{K}_{\mathrm{a}}=251 \mathrm{M}^{-1} \mathrm{~s}^{-1} ; \mathrm{K}_{\mathrm{d}}=2.64 \times 10^{-2} \mathrm{~s}^{-1}\right)$, bavachinMAPK1 $\left(\mathrm{K}_{\mathrm{a}}=9.29 \times 10^{3} \mathrm{M}^{-1} \mathrm{~s}^{-1} ; \mathrm{K}_{\mathrm{d}}=2.47 \times 10^{-1} \mathrm{~s}^{-1}\right)$, and liquiritin-MAPK1 $\left(\mathrm{K}_{\mathrm{a}}=258 \mathrm{M}^{-1} \mathrm{~s}^{-1} ; \mathrm{K}_{\mathrm{d}}=9.84 \times 10^{-2} \mathrm{~s}^{-1}\right)$ exhibit binding and/or release kinetics characteristics, which 
A

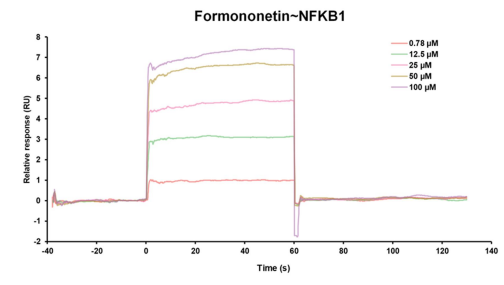

B

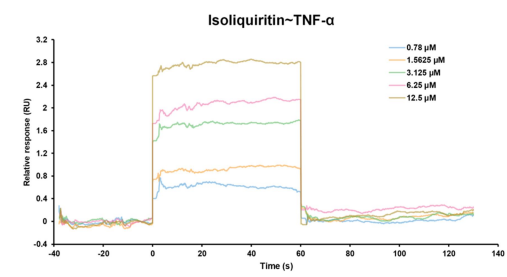

C

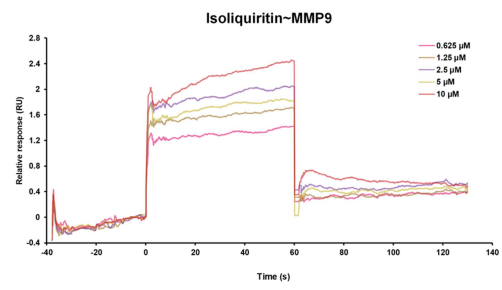

D

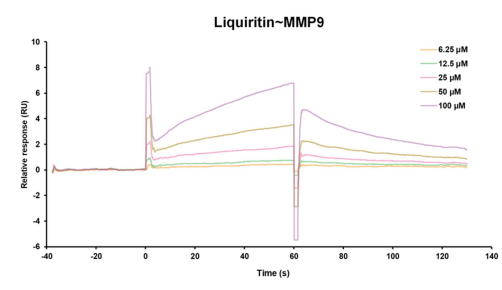

F

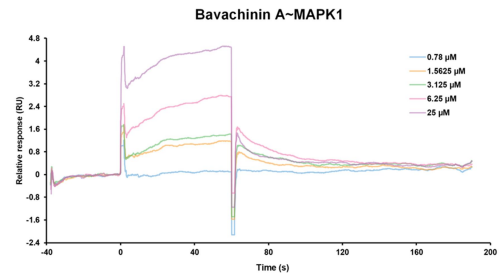

G

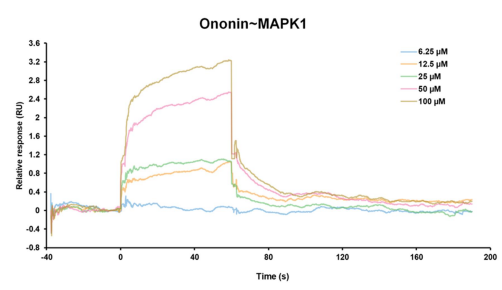

H

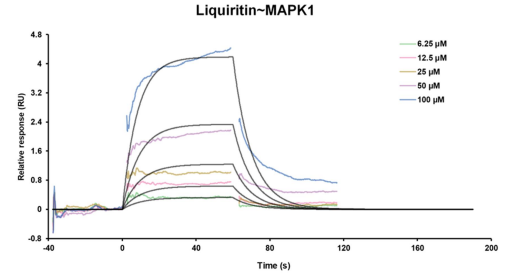

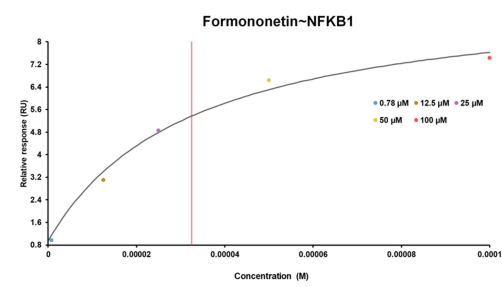
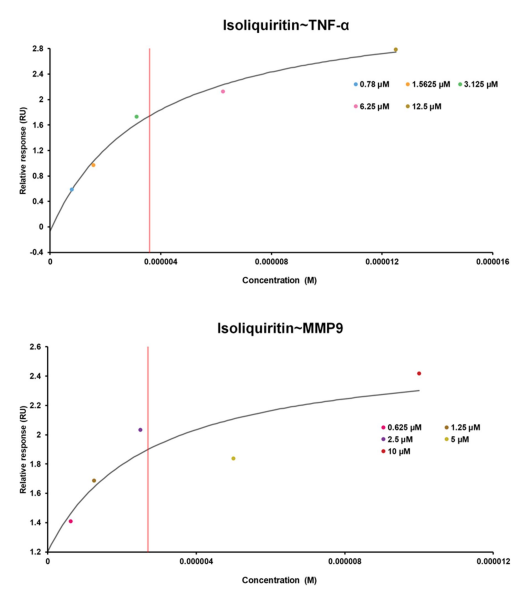

E
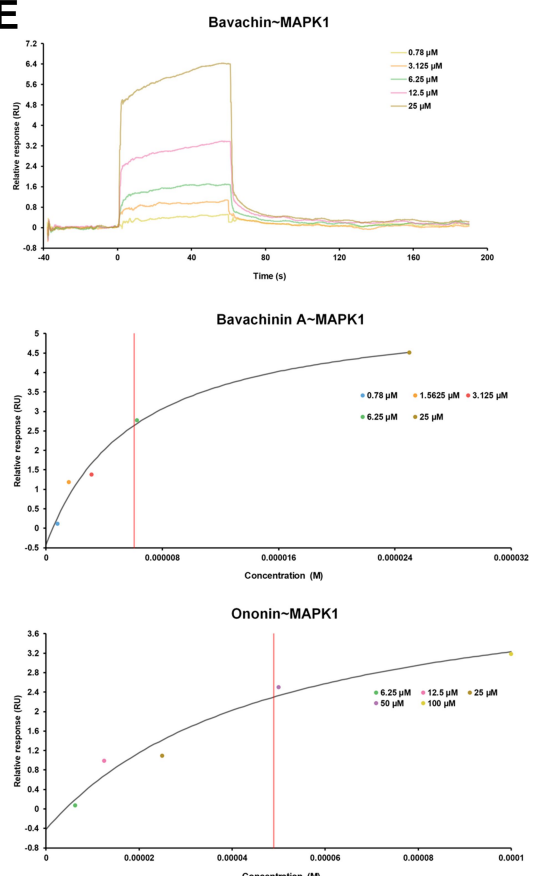

Figure 7 The surface plasmon resonance (SPR) assay of the interaction of formononetin with NFKBI, isoliquiritin with TNF- $\alpha$, MMP9 proteins, liquiritin with MMP9, and bavachin, bavachinin A, liquiritin, ononin with MAPKI. (A) The SPR curves of formononetin binding to NFKBI (left) and the representative binding curve (right). (B) The SPR curves of isoliquiritin binding to TNF- $\alpha$ (left) and the representative binding curve (right). (C) The SPR curves of isoliquiritin binding to MMP9 (left) and the representative binding curve (right). (D) The SPR curves of liquiritin binding to MMP9. (E) The SPR curves of bavachin binding to MAPKI. (F) The SPR curves of bavachinin A binding to MAPKI (left) and the representative binding curve (right). (G) The SPR curves of ononin binding to MAPKI (left) and the representative binding curve (right). (H) The SPR curves of liquiritin binding to MAPKI. 
A

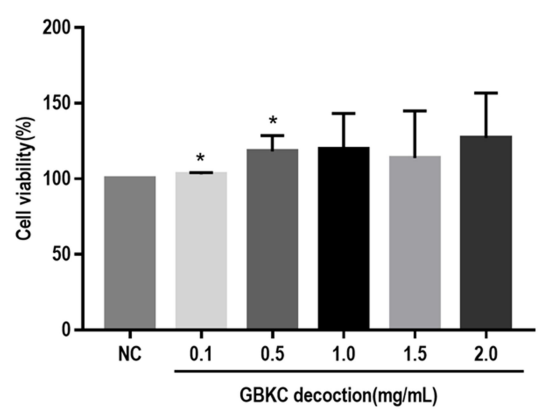

B

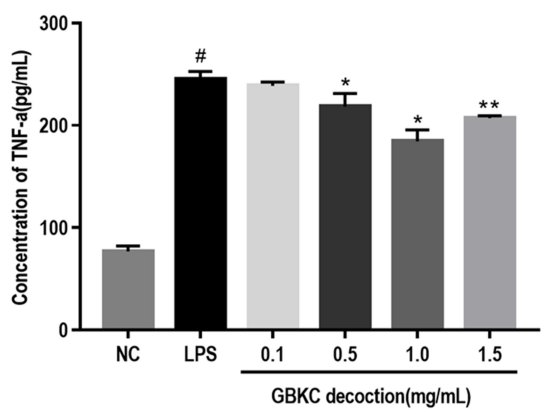

C

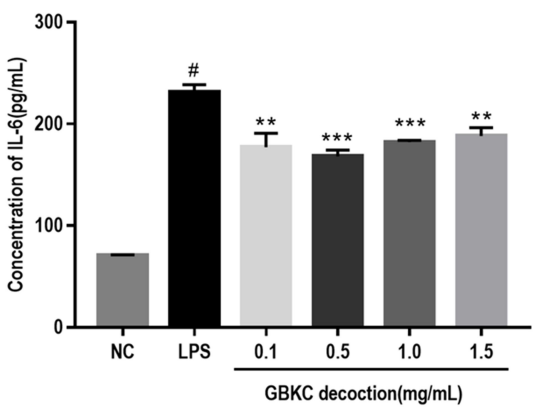

Figure 8 GBKC alleviates the secretions of IL- 6 and TNF- $\alpha$ in LPS-induced MH-S cells. (A) Cell viability. Data are presented as the mean \pm SD ( $=3$ ) $* p<0.05$, compared with normal control (NC) group. (B) TNF- $\alpha$. (C) IL-6. Data are presented as the mean \pm SD $(n=3) \# p<0.000$ I compared with NC group. *p< 0.05 ; **p< 0.005 ; *** $p<$ 0.0005 , compared with the LPS group.

may provide valuable information for further lead compound discovery. Collectively, these results demonstrate the validity of network pharmacology and molecular docking approaches, and the in-depth biological functions of these ligand-target pairs are worth exploring.

\section{Effect of GBKC on TNF- $\alpha$ and IL-6 Protein Release After LPS Induction}

CCK-8 assay indicated that no obvious cytotoxicity to $\mathrm{MH}-$ $\mathrm{S}$ cells was noted after GBKC treatment at the chosen concentrations (Figure 8A). As shown in Figure $8 \mathrm{~B}$ and $\mathrm{C}$, the levels of TNF- $\alpha$ and IL-6 were markedly increased in $\mathrm{MH}-\mathrm{S}$ cells after LPS induction. However, GBKC administration caused statistically significant reductions in TNF- $\alpha$ and IL-6 secretion in a dose-dependent manner. The results also indicated that GBKC exhibited the best inhibitory effects on TNF- $\alpha$ at $1 \mathrm{mg} / \mathrm{mL}$ and IL- 6 at $0.5 \mathrm{mg} / \mathrm{mL}$.

\section{Conclusions}

TCM has accumulated valuable information regarding alleviation of $\mathrm{CB}$ progression. In the present study, 53 main components were identified from GBKC using UHPLCLTQ-Orbitrap. According to the identified compounds, network and pathway analyses indicated that the TNF signaling pathway was closely related to 13 key targets (TNF- $\alpha, \mathrm{NF} \kappa \mathrm{B}$, I $\mathrm{B} \alpha$, MAPK1, PIK3CA, PIK3R1, PIK3R2, PIK3R3, MMP9, PTGS2, IL-1 $\beta$, IL-6, and CCL2) and the anti-CB effect of GBKC. In vitro experiments demonstrated that GBKC inhibited TNF- $\alpha$ and IL- 6 release in a dose-dependent manner. Furthermore, molecular docking and SPR assays showed that several constituents exhibited good affinity to specific targets (formononetin-NFKB1, $\mathrm{K}_{\mathrm{D}}=32.5 \mu \mathrm{M}$; isoliquiritin-TNF- $\alpha$, $\mathrm{K}_{\mathrm{D}}=3.6 \mu \mathrm{M}$; isoliquiritin-MMP-9, $\mathrm{K}_{\mathrm{D}}=2.7 \mu \mathrm{M}$; bavachinin A-MAPK $\left.1, K_{D}=6.05 \mu \mathrm{M}\right)$. This study provides a systematic perspective to determine the anti-CB mechanisms of GBKC and also offers a novel approach to illustrate the chemical and pharmacological basis of other herbal medicines. Further experiments are required to validate these hypotheses to enhance the reliability of the results.

\section{Acknowledgments}

We would like to thank Lei Zhang and Wenning Yang for discussions and technical assistance.

\section{Funding}

This study was supported by the National Key Research and Development Program of China (No. 2019YFC 1710105).

\section{Disclosure}

The authors declared no conflict of interests for this work.

\section{References}

1. Valipour A, Fernandez-Bussy S, Ing AJ, et al. Bronchial rheoplasty for treatment of chronic bronchitis. twelve-month results from a multicenter clinical trial. Am J Respir Crit Care Med. 2020;202 (5):681-689. doi:10.1164/rccm.201908-1546OC

2. Chan SMH, Selemidis S, Bozinovski S, Vlahos R. Pathobiological mechanisms underlying metabolic syndrome (MetS) in chronic obstructive pulmonary disease (COPD): clinical significance and therapeutic strategies. Pharmacol Ther. 2019;198:160-188.

3. Weiss A, Porter S, Rozenberg D, et al. Chronic obstructive pulmonary disease: a palliative medicine review of the disease, its therapies, and drug interactions. J Pain Symptom Manage. 2020;60(1):135-150. doi:10.1016/j.jpainsymman.2020.01.009

4. Amjad E, Asnaashari S, Sokouti B. The role of associated genes of Wnt signaling pathway in chronic obstructive pulmonary disease (COPD). Gene Rep. 2020;18:100582. doi:10.1016/j.genrep.2019.100582

5. Gonçalves PB, Romeiro NC. Multi-target natural products as alternatives against oxidative stress in Chronic Obstructive Pulmonary Disease (COPD). Eur J Med Chem. 2019;163:911-931. doi:10.1016/ j.ejmech.2018.12.020 
6. Hanania NA, Chapman KR, Kesten S. Adverse effects of inhaled corticosteroids. Am J Med. 1995;98(2):196-208. doi:10.1016/S00029343(99)80404-5

7. Chinese Pharmacopoeia Commission. Pharmacopoeia of the People's Republic Of China. Beijing, China: People's Medical Publishing House; 2020.

8. Wang W, Sun Z, Xu L, Zhu X. Effect of Guben Kechuan Granules on expression of thymus index and spleen index of rats with experimental chronic obstructive pulmonary disease. Chin $J$ Clin Pharmacol. 2015;31(11):935-937.

9. Wang W, Sun Z, Xu L, Zhu X. Effect of Guben Kechuan granules on expression of pathology and $\mathrm{T}$ lymphocyte subsets in rats with chronic obstructive pulmonary disease. Chin J Clin Pharmacol. 2015;31(10):840-842.

10. Wang W, Sun Z, Xu L, Zhu X. Effect of Guben Kechuan Granules on expression of soluble interleukin-2 receptor of rats with chronic obstructive pulmonary disease. Chin J Clin Pharmacol. 2015;31(01):28-30.

11. Luo Z, Yu G, Chen X, et al. Integrated phytochemical analysis based on UHPLC-LTQ-Orbitrap and network pharmacology approaches to explore the potential mechanism of Lycium ruthenicum Murr. for ameliorating Alzheimer's disease. Food Funct. 2020;11(2):13 62-1372. doi:10.1039/C9FO02840D

12. Yu G, Luo Z, Zhou Y, et al. Uncovering the pharmacological mechanism of Carthamus tinctorius L. on cardiovascular disease by a systems pharmacology approach. Biomed Pharmacother. 2019;117:109094. doi:10.1016/j.biopha.2019.109094

13. Wang W, Liu T, Yang L, et al. Study on the multi-targets mechanism of triphala on cardio-cerebral vascular diseases based on network pharmacology. Biomed Pharmacother. 2019;116:108994. doi:10.10 16/j.biopha.2019.108994

14. Qin H, Chen H, Zou Y, et al. Systematic investigation of the mechanism of Cichorium glandulosum on type 2 diabetes mellitus accompanied with non-alcoholic fatty liver rats. Food Funct. 2019;10 (5):2450-2460. doi:10.1039/C8FO02284D

15. Jing C, Sun Z, Xie X, et al. Network pharmacology-based identification of the key mechanism of Qinghuo Rougan Formula acting on uveitis. Biomed Pharmacother. 2019;120:109381. doi:10.1016/j. biopha.2019.109381

16. Taha KF, Khalil M, Abubakr MS, Shawky E. Identifying cancer-related molecular targets of Nandina domestica Thunb. by network pharmacology-based analysis in combination with chemical profiling and molecular docking studies. $J$ Ethnopharmacol. 2020;249:112413. doi:10.1016/j.jep.2019.112413

17. Zhao M, Chen Y, Wang C, et al. Systems Pharmacology Dissection of Multi-Scale Mechanisms of Action of Huo-Xiang-Zheng-Qi Formula for the Treatment of Gastrointestinal Diseases. Front Pharmacol. 2019;9:1448. doi:10.3389/fphar.2018.01448
18. Li R, Li Q, Ji Q. Molecular targeted study in tumors: from western medicine to active ingredients of traditional Chinese medicine. Biomed Pharmacother. 2020;121:109624. doi:10.1016/j.biopha.20 19.109624

19. He Q, Chen Y, Shen D, et al. Development of a surface plasmon resonance immunosensor and ELISA for 3-nitrotyrosine in human urine. Talanta. 2019;195:655-661. doi:10.1016/j.talanta.2018.11.110

20. Liu X, Luo F, Li P, She Y, Gao W. Investigation of the interaction for three Citrus flavonoids and $\alpha$-amylase by surface plasmon resonance. Food Res Int. 2017;97:1-6. doi:10.1016/j.foodres.2017.03.023

21. Yang L, Hou A, Wang S, et al. Screening and quantification of TNF- $\alpha$ ligand from Angelicae Pubescentis Radix by biosensor and UPLCMS/MS. Anal Biochem. 2020;596:113643. doi:10.1016/j.ab.2020.11 3643

22. Chen L, Wang D, Lv D, et al. Identification of eupatilin and ginkgolide $\mathrm{B}$ as p38 ligands from medicinal herbs by surface plasmon resonance biosensor-based active ingredients recognition system. J Pharm Biomed Anal. 2019;171:35-42. doi:10.1016/j.jpba.2019. 03.029

23. Yu G, Zhang Y, Ren W, et al. Network pharmacology-based identification of key pharmacological pathways of Yin-Huang-Qing-Fei capsule acting on chronic bronchitis. Int J Chron Obstruct Pulmon Dis. 2016;12:85-94. doi:10.2147/COPD.S121079

24. Corinna K, Martin HA, Marc Z, Juliane F. Identification of new drug classification terms in textual resources. Bioinformatics. 2007;23(13): i264-i272. doi:10.1093/bioinformatics/btm196

25. Hamosh A, Scott AF, Amberger JS, Bocchini CA, McKusick VA. Online Mendelian Inheritance in Man (OMIM), a knowledgebase of human genes and genetic disorders. Nucleic Acids Res. 2005;33 (Database issue):D514-D517. doi:10.1093/nar/gki033

26. Jensen LJ, Kuhn M, Stark M, et al. STRING 8-a global view on proteins and their functional interactions in 630 organisms. Nucleic Acids Res. 2009;37(Database issue):D412-D416. doi:10.1093/nar/ gkn760

27. Wang J, Gao Y, Lin F, Han K, Wang X. Omentin-1 attenuates lipopolysaccharide (LPS)-induced U937 macrophages activation by inhibiting the TLR4/MyD88/NF- $\mathrm{BB}$ signaling. Arch Biochem Biophys. 2020;679:108187. doi:10.1016/j.abb.2019.108187

28. Matera MG, Calzetta L, Cazzola M. TNF- $\alpha$ inhibitors in asthma and COPD: we must not throw the baby out with the bath water. Pulm Pharmacol Ther. 2010;23(2):121-128. doi:10.1016/j.pupt.2009.10. 007

29. Di Francia M, Barbier D, Mege JL, Orehek J. Tumor necrosis factor-alpha levels and weight loss in chronic obstructive pulmonary disease. Am J Respir Crit Care Med. 1994;150(5 Pt 1):1453-1455. doi:10.1164/ajrccm.150.5.7952575
Journal of Inflammation Research

\section{Publish your work in this journal}

The Journal of Inflammation Research is an international, peerreviewed open-access journal that welcomes laboratory and clinica findings on the molecular basis, cell biology and pharmacology of inflammation including original research, reviews, symposium reports, hypothesis formation and commentaries on: acute/chronic inflammation; mediators of inflammation; cellular processes; molecular mechanisms; pharmacology and novel anti-inflammatory drugs; clinical conditions involving inflammation. The manuscript management system is completely online and includes a very quick and fair peerreview system. Visit http://www.dovepress.com/testimonials.php to read real quotes from published authors. 